\title{
A FORMULA FOR SEMIGROUPS, WITH AN APPLICATION TO BRANCHING DIFFUSION PROCESSES
}

\author{
BY \\ STANLEY A. SAWYER( $\left.{ }^{(}\right)$
}

\begin{abstract}
A Markov process $P=\left\{x_{t}\right\}$ proceeds until a random time $\tau$, where the distribution of $\tau$ given $P$ is $\exp \left(-\phi_{t}\right)$ for finite additive functional $\left\{\phi_{t}\right\}$, at which time it jumps to a new position given by a substochastic kernel $K\left(x_{\tau}, A\right)$. A new time $\tau^{\prime}$ is defined, the process again jumps at a time $\tau+\tau^{\prime}$ and so forth, producing a new Markov process $\boldsymbol{P}^{\prime}$. A formula for the infinitesimal generator of the new process (in terms of the i.g. of the old) is then derived. Using branching processes and local times $\left\{\phi_{t}\right\}$, classical solutions of some linear partial differential equations with nonlinear boundary conditions are constructed. Also, conditions are given guaranteeing that a given Markov process is of type $P^{\prime}$ for some triple $\left(P,\left\{\phi_{t}\right\}, K\right)$.
\end{abstract}

1. Introduction. Let $P$ be a Markov process on a metric state space $X$. We construct a new process $P^{\prime}$ as follows. We proceed according to the process $P$ until a random time given by a random "clock" (see $\S 2$ for precise definitions), at which time we stop the process and restart it at a new position given by a substochastic kernel $K(x, A)$ (where $x$ refers to the position of $P$ at the random time). We reset the "clock" and proceed from the new position until a new random time, "jump" a second time, reset the clock, and so forth. The purpose of this paper is to derive an expression for the infinitesimal generator of (the semigroup of) the process $P^{\prime}$ in terms of the infinitesimal generator of $P$, the kernel $K(x, A)$ and the additive functional running the clock. (See equations (2.7), (2.8) below.) As an example, using branching processes and local times, we construct classical solutions of some linear partial differential equations with nonlinear boundary conditions.

The process $P^{\prime}$ described above was first considered in a general setting by Moyal [13]. He worked with the transition function of $P$, and obtained an equation for the transition function of $P^{\prime}$. This equation, essentially the equation (2.5) below, was shown always to have at least one solution, and the meaning of nonuniqueness was discussed. These questions were given considerable impetus in 1964 by Skorohod [19] (and Itô-McKean [7]) who showed that a process of type $P^{\prime}$ could be used to give solutions of certain semilinear partial differential equations of parabolic type. A probabilistic construction of the process $P^{\prime}$ was carried out in [6] (see also [5],

Received by the editors June 27, 1969.

AMS 1969 subject classifications. Primary 6060, 6067, 3565; Secondary 6062, 3562.

Key words and phrases. Markov processes, diffusion processes, semigroups, branching processes, infinitesimal generator.

(1) The author was partially supported by NSF grant GP-8975.

Copyright (C) 1970, American Mathematical Society 
[18], [16]). There a model of the process $P^{\prime}$ was built by a brute force procedure of "cutting and pasting" countably many copies of the process $P$, which showed among other things that the strong Markov property would not be destroyed. The set-up in that construction was essentially that of the first paragraph, except that the kernel $K(x, A)$ could depend on the "tail-field" of $P$ before the random time and not just its position. In the special case when the additive functional running the clock is of "Kac type" (see (2.16) below), a formula for the infinitesimal generator of $P^{\prime}$ was then (in essence) derived. In a branching diffusion context this gave a complete probabilistic treatment of exactly the class of equations considered by [19] and [7].

The purpose here is to generalize the class of additive functionals beyond those of Kac type to include "local times." The use of a local time in a partial differential equation setting would lead to the introduction of a nonlinear boundary condition rather than the introduction of a nonlinear potential term in the equation. In particular, it would involve a change in the domain of the infinitesimal generator rather than in its actual values; in contrast we remark that all previous investigations of the infinitesimal generator of $P^{\prime}$ have contained assumptions requiring the domains of the infinitesimal generators to be the same.

2. Statement of results. Let $P=\left\{x_{t}, B_{t}\right\}$ be a strong Markov process with respect to the $\sigma$-algebras $B_{t}=\bigcap_{\varepsilon \varepsilon>0]} B\left[\left\{x_{s}: s \leqq t+\varepsilon\right\}\right]$, which we assume has rightcontinuous paths in a metric state space $X$ and transition function $P(t, x, A)$ (see e.g. [2, Chapter 3]). Let $K(x, A)$ be a substochastic kernel on $X$, i.e. (i) for fixed $x \in X, K(x, A)$ is a nonnegative Borel measure on $X$ with $K(x, X) \leqq 1$, and (ii) for a fixed Borel set $A \subseteq X, K(x, A)$ is a Borel function on $X$. This last condition insures that the operator $K f(x)=\int f(y) K(x, d y)$ preserves the class of bounded Borel functions on $X$ which, with a convenient abuse of notation, we denote by $\mathscr{L}^{\infty}(X)$. As is standard, we assume the existence of an "escape point" $\Delta \notin X$ in order to have a probabilistic interpretation of $P(t, x, X)<1$ or $K(x, X)<1$; i.e. at some "termination time" $\zeta(\omega)$ the process $P$ escapes to and is trapped at $\Delta$. $P(t, x, X)$ is then the probability that the process is still in $X$. The actual state space is then always understood to be $X \cup\{\Delta\}$; unless otherwise indicated, all $f(x) \in \mathscr{L}^{\infty}(X)$ will be assumed to be defined on $X \cup\{\Delta\}$ and satisfy $f(\Delta)=0$.

Let $\left\{\phi_{t}(\omega)\right\}$ be a (finite) additive functional of the process $P$, i.e. a collection of random variables measurable on the process such that

(i) $\phi_{t}(\omega)$ is $B_{t}$-measurable for all $t$,

(ii) $\phi_{0}(\omega) \equiv 0,0 \leqq \phi_{t}(\omega)<\infty$,

(iii) $\phi_{t+s}(\omega)=\phi_{t}(\omega)+\theta_{t} \phi_{s}(\omega)$,

(iv) $\left\{\phi_{t}(\omega)\right\}$ is right continuous in $t$ for all $\omega$,

where " $\theta_{t}$ " is the time-shift operator of Dynkin. In particular, $\phi_{t}(\omega) \uparrow$ for all $\omega$. We also assume $\phi_{t}(\omega) \equiv \phi_{\zeta(\omega)-}(\omega)$ for $t \geqq \zeta(\omega)$; this is equivalent to assuming 
$E_{\Delta}\left(\phi_{t}\right) \equiv 0$ and $P_{x}\left(\phi_{s}-\phi_{s-}>0, s=\zeta\right) \equiv 0$. In addition, we will from time to time use $\left\{\phi_{t}(\omega)\right\}$ is continuous in $t$ for all $\omega$.

Examples would be "local times," additive functionals of "Kac type" (see (2.16) below), or additive functionals of saltus type which change only by jumps at the jumps of $\left\{x_{t}\right\}$. The random time of $\S 1$, when the process is required to jump according to $K(x, A)$, is defined as follows (using an idea of Hunt): Let $m$ be a random variable independent of the process $\left\{x_{t}\right\}$ with $P_{x}(m>t)=e^{-t} ; m$ can be constructed, if need be, by extending the probability space. The random time is then

$$
\tau(\omega)=\sup \left\{t: \phi_{t}(\omega) \leqq m\right\}=\phi_{m}^{-1}(\omega),
$$

i.e. the right-continuous inverse of $\phi_{t}(\omega)$ at $m$. If $B_{\infty}=\bigcup_{t} B_{t}$, then

$$
P_{x}\left(\tau>t / B_{\infty}\right)=P_{x}\left(\phi_{t} \leqq m / B_{\infty}\right)=e^{-\phi_{t}} \text { a.s. }
$$

Let $P^{\prime}=\left\{x_{t}^{\prime}, B_{t}^{\prime}\right\}$ be the process described in $\$ 1$ (which we define to be at $\Delta$ after a finite time accumulation point of jumps). Set $T_{t} f(x)=E_{x}\left(f\left(x_{t}\right)\right)=\int f(y) P(t, x, d y)$, $T_{t}^{\circ} f(x)=E_{x}\left(f\left(x_{t}\right) \chi_{(\tau>t)}\right)=E_{x}\left(f\left(x_{t}\right) e^{-\phi_{t}}\right)$ and $T_{t}^{\prime}(f(x))=E_{x}^{\prime}\left(f\left(x_{t}^{\prime}\right)\right)$. Then $\left\{T_{t}^{\circ}\right\}$ and $\left\{T_{t}^{\prime}\right\}$ are semigroups, and $\bar{T}_{t}=T_{t}^{\prime}$ is a solution of the equation

$$
\bar{T}_{t} f(x)=T_{t}^{\circ} f(x)+E_{x}\left(\chi_{(\tau \leqq t)} \int_{X} \bar{T}_{t-\tau} f(y) K\left(x_{\tau}, d y\right)\right)
$$

for all $f(x) \in \mathscr{L}^{\infty}(X)$ (see [2, Chapter 9], [6], [13]). Note that formally, (2.5) is just the strong Markov property applied at $\tau(\omega)$. (Let $f(x)=\chi_{A}(x)$ be the characteristic function of $A$; then $T_{t} f(x)=P(t, x, A), T_{t}^{\circ} f(x)=P_{x}\left(x_{t} \in A, \tau>t\right)$, etc. $)$

Finally a word about the domain of the semigroups involved. By standard (weak) semigroup theory (e.g. [2, Chapter 1]), $\left\{T_{t}\right\}$ is naturally defined on

$$
B_{0}=\left\{f(x): f(x) \in \mathscr{L}^{\infty}(X), \lim _{t \rightarrow 0} T_{t} f(x)=f(x) \text { for all } x\right\} .
$$

Condition 2.1(iv) guarantees that $B_{0}$ is the same if $\left\{T_{t}\right\}$ is replaced by $\left\{T_{t}^{\circ}\right\}$ or any Markov semigroup $\left\{\bar{T}_{t}\right\}$ on $X$ satisfying (2.5). In general, we let $B$ be any linear subspace of $B_{0}$ which is preserved by the six families of operators $T_{t}, T_{t}^{\circ}, \bar{T}_{t}$, $R_{\lambda}, R_{\lambda}^{\circ}, \bar{R}_{\lambda}$, where the $R_{\lambda}$ 's are the resolvent (Laplace transform) operators of the corresponding semigroups $(\lambda>0)$. The space $B_{0}$ always satisfies this condition; of greater interest in application is the fact that if $\left\{T_{t}\right\}$ is strongly Feller and $P_{x}(\tau \leqq t)$ $=\sigma(1)$ uniformly in $x$, then $\left\{T_{t}^{\circ}\right\}$ and $\left\{\bar{T}_{t}\right\}$ are strongly Feller (see Theorem 5.3 in $\S 5$ ) and $B=B C(X)$ (the space of bounded continuous functions on $X$ ) will be preserved by these six operators. With some $B$ fixed, we define $A, A^{\circ}, \bar{A}$ as the (weak) infinitesimal generators of the three semigroups on $B$, with domains (for example for $A$ )

$$
\begin{aligned}
\mathscr{D}(A)=\{f(x): & f(x) \in B,\left|(1 / t)\left(T_{t} f(x)-f(x)\right)\right| \leqq M, \\
& \left.(1 / t)\left(T_{t} f(x)-f(x)\right) \rightarrow \text { some } g(x) \in B, \text { all } x\right\}
\end{aligned}
$$

(which is also the range of $R_{\lambda}$ on $B$ ) with $A f(x)=g(x)$. Set $A_{\lambda}=A-\lambda I, A_{\lambda}^{\circ}=A^{\circ}-\lambda I$ etc. for $\lambda>0$; thus $A_{\lambda}$ maps $\mathscr{D}(A)$ onto $B$ and $A_{\lambda} R_{\lambda} \equiv-I$. 
It will be noted that we are considering the "weak" infinitesimal generator and not the "strong" generator of the Hille-Yosida theorems. This is partly a matter of taste; the weak generator seems more natural probabilistically. However if $T_{t} f(x), T_{t}^{\circ} f(x), \bar{T}_{t} f(x) \rightarrow f(x)$ uniformly for all $f(x) \in B$, the weak and strong infinitesimal generators would coincide on $B$, and what follows would hold a posteriori for the strong generator as well.

We are now ready to state our main results, which depend, if $\left\{\phi_{t}(\omega)\right\}$ is continuous in $t$, on the operators

$$
S_{\lambda} f(x)=E_{x}\left[\int_{0}^{\infty} e^{-\lambda s} f\left[x_{s}(\omega)\right] d \phi_{s}(\omega)\right]
$$

for nonnegative $f(x) \in \mathscr{L}^{\infty}(X)$. If (2.2) does not hold, the results to follow are valid provided $S_{\lambda}$ is replaced by

$$
S_{\lambda} f(x)=-E_{x}\left(\int_{0}^{\infty} e^{-\lambda s} f\left[x_{s}(\omega)\right] \exp \left[\phi_{s_{-}}(\omega)\right] d\left(\exp \left[-\phi_{s}(\omega)\right]\right)\right) .
$$

THEOREM 2.1. Let $\left\{\phi_{t}(\omega)\right\}$ satisfy (2.1), and assume $\left\{\bar{T}_{t}\right\}$ is some Markovian semigroup on $X$ which satisfies (2.5). Assume further that $S_{\lambda} 1(x)<\infty$ for all $x$ and some $\lambda>0$. Then

$$
\bar{A}_{\lambda} \subseteq A_{\lambda}\left(I-S_{\lambda}(K-I)\right)
$$

where the right-hand side of $(2.8)$ is interpreted as a linear operator whose domain is the set of all $f(x) \in B$ with $f-S_{\lambda}(K-I) f \in \mathscr{D}(A)$.

A version of Theorem 2.1 where $\lambda=0$ is stated in Theorem 2.4. The question of equality in (2.8) (i.e. when $f \in \mathscr{D}(\bar{A})$ iff $\left.f-S_{\lambda}(K-I) f \in \mathscr{D}(A)\right)$ is important as it gives a sufficient condition for $f(x) \in \mathscr{D}(\bar{A})$. This, in turn, is closely connected with the question of uniqueness of solutions of (2.5). (See $\$ 4$ for proofs of Theorems 2.2, 2.4.)

THEOREM 2.2. If $B=B_{0}$, the inclusion (2.8) is attained for any solution $\left\{\bar{T}_{t}\right\}$ of (2.5) iff there exists a unique Markovian semigroup solution of (2.5), which occurs iff there exists no nontrivial bounded measurable solutions of the equation

$$
f(x)=E_{x}\left(e^{-\lambda \tau} \int f(y) K\left(x_{\tau}, d y\right)\right) .
$$

For a general B, the inclusion (2.8) is attained (in B) iff (2.9) has no nontrivial solutions $f(x) \in B$, either of which implies that (2.5) has a unique solution $\left\{\bar{T}_{t}\right\}$ subject to $\left\{\bar{T}_{t}\right\}$ and $\left\{\bar{R}_{\lambda}\right\}$ preserving $B$. A sufficient (but not necessary) condition for equality in (2.8) etc. (for any $B$ ) is either of

$$
\begin{array}{ll}
\text { (i) } P_{x}\left(\tau \leqq t_{0}\right) \leqq \alpha<1, & \text { some } t_{0}>0 \text {, all } x \text {, } \\
\text { (ii) } E_{x}\left(\phi_{t_{0}}\right) \leqq C<\infty, & \text { some } t_{0}>0 \text {, all } x .
\end{array}
$$

Condition (2.9) (and its proof) are actually generalizations of Feller's work on continuous-time Markov chains [3]. (See e.g. Theorem 7.7.5, p. 368, in [24].) As 
in Moyal [13] uniqueness fails in Theorem 2.2 iff the successive "random jumping times" of $\S 1$ have a finite upper bound with positive probability. Nonuniqueness results from the fact that $(2.5)$ only prescribes the behavior of the process $P^{\prime}$ up to such an "explosion" time; at such a time the process could jump anywhere it likes without affecting (2.5). The question of when finite explosion times can occur with positive probability (at least when $\left.K(x, A)=\delta_{x}(A)\right)$ is considered in greater detail in \$3.1.

For branching Markov processes these results take the following form (see $\$ 6$ for details). The term "branching Markov process" here will refer to the same set-up as in $\S 1$, except that at the random time the process $P$ may branch into multiple copies of itself at various locations, which then proceed independently of one another and of their parents. Each has its own $\tau$ independently of other particles, and eventually branches into daughters of its own (leaving sisters and aunts undisturbed), etc. If $P=\left\{x_{t}, B_{t}\right\}$ is a process as before on a metric state space $D$, the new process will live in the space $X=\cup_{0}^{\infty} D^{n}=\{\partial\} \cup D \cup D \times D \cup \cdots$, where $D^{n}$ refers to possible distributions of $n$ existing particles and $\partial$ refers to the extinction of all particles (i.e. all die childless).

The process $\bar{P}$ on $X$ is constructed (see $\S 6$ ) from the process $P$ (on $D$ ), the branching time distribution $\left\{\phi_{t}\right\}$ (via (2.3)) and branching location distributions $\left\{\pi_{0}(a)\right.$, $\left.\pi_{n}(a, E)\right\}\left(a \in D, E \subseteq D^{n}\right)$ for a single particle. We define a nonlinear operator $\mathscr{L}$ for $f(a) \in \mathscr{L}^{\infty}(D),|f(a)| \leqq 1$, by

$$
\begin{aligned}
\mathscr{L} f(a) & =\sum_{0}^{\infty} \int_{D^{n}} \hat{f}(y) \pi_{n}(a, d y) \\
& =\pi_{0}(a)+\int_{D} f(b) \pi_{1}(a, d b)+\int_{D} \int_{D} f\left(b_{1}\right) f\left(b_{2}\right) \pi_{2}\left(a, d b_{1} x d b_{2}\right)+\cdots
\end{aligned}
$$

where $\hat{f}(y)$ is defined by (6.8). By construction $\mathscr{L} 1(a) \equiv 1$; if for example $\pi_{n}(a, E)$ $=p_{n}(a) \delta_{(a, a, \cdots, a)}(E)$, then $\mathscr{L} f(a)=\sum p_{n}(a) f(a)^{n}$. Let $\bar{A}$ be the weak infinitesimal generator of $\bar{P}$ on $X$ (with $B=B_{0}$ for simplicity) and $A$ the generator of $P$ on $D$ (again with maximal domain). Then

THEOREM 2.3 (see $\$ 7$ for proof). Assume $\left\{\phi_{t}\right\}$ satisfies (2.1), and that $E_{a}\left(\phi_{t}\right) \leqq C_{t}$ for all $a \in D$, where $C_{t} \downarrow 0$. Then

$$
\bar{A}_{\lambda} \subseteq A_{\lambda}\left(I-S_{\lambda}(\mathscr{L}-I)\right) \text { in } D,
$$

in the sense that if $\hat{f}(x) \in \mathscr{D}(\bar{A})$, then $f(a)-S_{\lambda}(\mathscr{L}-I) f(a) \in \mathscr{D}(A)$ on $D$ and $\bar{A}_{\lambda} \hat{f}(a)$ can be calculated by (2.12). Moreover, the inclusion (2.12) is attained for $|f(a)| \leqq c<1$. That is, if $|f(a)| \leqq c<1$, then $f(a)-S_{\lambda}(\mathscr{L}-I) f(a) \in \mathscr{D}(A)$ on $D$ iff $\hat{f}(x) \in \mathscr{D}(\bar{A})$ on $X$.

Corollary. Assume $f(a) \in \mathscr{L}^{\infty}(D)$ satisfies $|f(a)| \leqq c<1$ and $f(a)-S_{\lambda}(\mathscr{L}-I) f(a)$ $\in \mathscr{D}(A)$, and define

$$
u(a, t)=\bar{E}_{a}\left(\prod f\left(x_{t}^{(i)}\right)\right)
$$


where the product is over the (random) number of particles alive at time $t$ which are descendents of a single particle initially at $a$. Then, $u(a, t)$ is the unique regular solution (i.e. $u(a, t)-S_{\lambda}(\mathscr{L}-I) u(a, t) \in \mathscr{D}(A)$ for all $t>0$ with $\left.|u(a, t)| \leqq c^{\prime}<1\right)$ of the formally nonlinear equation

$$
\left(\partial^{+} / \partial t-\lambda\right) u(a, t)=A_{\lambda}\left(I-S_{\lambda}(\mathscr{L}-I)\right) u(a, t)
$$

with $u(a, 0)=f(a)$, where " $\partial^{+} / \partial t$ " is a one-sided derivative.

Proof. Evidently $u(a, t)=\bar{T}_{t} \hat{f}(a)$, where $\hat{f}(x) \in \mathscr{D}(\bar{A})$. For uniqueness, use the product rule to extend (2.14) to $X$ and apply Theorem 2.3 and standard semigroup arguments in $X$.

That we can get equality in (2.12) in any sense might be unexpected, since even with $E_{a}\left(\phi_{t}\right) \leqq C_{t} \downarrow 0$ in $D$ it is possible to have "finite explosion times" and definitely possible to have multiple semigroup solutions of the equation in $X$ corresponding to (2.5) or nontrivial solutions of the analogue of (2.9). However, if we assume for example

$$
\sum_{0}^{\infty} n \pi_{n}\left(a, D^{n}\right) \leqq Q<\infty, \quad a \in D,
$$

in combination with $E_{a}\left(\phi_{t}\right) \leqq C_{t} \downarrow 0$, then by standard arguments we can exclude "finite explosion times" (or nonuniqueness in $X$ ) and conclude equality in (2.12) for $|f(a)| \leqq 1$.

We have the following version of Theorem 2.1 for $\lambda=0$; similar arguments could also be made for branching processes. See $\S 4$ for proof.

TheOREM 2.4. Assume $\left\{\phi_{t}\right\}$ satisfies (2.1), and also that $E_{x}(\zeta) \leqq C<\infty$. Let $\left\{\bar{T}_{t}\right\}$ be a Markovian semigroup solution of (2.5), and let $S f(x)=S_{0} f(x)=\lim _{\lambda \rightarrow 0} S_{\lambda} f(x)$ in (2.7). Then if $S|f|(x)<\infty$ and $S K|f|(x)<\infty$ for all $x$ and $f \in \mathscr{D}(\bar{A})$,

$$
\bar{A} \subseteq A(I-S(K-I)) \text {. }
$$

If $\bar{E}_{x}(\zeta) \leqq C$ for some solution of (2.5), then the three "iff" statements carry over from Theorem 2.2 with $\lambda$ replaced by zero. A sufficient condition for $\bar{E}_{x}(\zeta) \leqq C$, as well as equality in (2.15) etc., is (2.2) combined with one of the conditions (2.10).

EXAmples. (1) Assume $\left\{\phi_{t}\right\}$ is of "Kac type," i.e.

$$
\phi_{t}(\omega)=\int_{0}^{t} V\left[x_{s}(\omega)\right] d s, \quad 0 \leqq V(x) \leqq C,
$$

where $f(x) \in B$ implies $V(x) f(x)$ and $K f(x) \in B$. For example, if $B=B C(X)$, if $V(x) \in B C(X)$ and $K$ is Feller, then $S_{\lambda} f(x)=R_{\lambda}(V f)(x)$ by (2.7), (2.16), and (2.10) is satisfied since $E_{x}\left(\phi_{t}\right) \leqq C t$. Hence by Theorems 2.1, 2.2

$$
\begin{aligned}
\bar{A}_{\lambda} & =A_{\lambda}\left(I-S_{\lambda}(K-I)\right)=A_{\lambda}-A_{\lambda} R_{\lambda} V(K-I), \\
\bar{A} & =A+V(K-I), \quad \mathscr{D}(A)=\mathscr{D}(\bar{A}),
\end{aligned}
$$


which has been (essentially) obtained by [6], [18], J. Haezendonck (personal communication) and perhaps others (it is even implicitly in Moyal [13, Theorem 4.2]). On the other hand, if $B=B C(X), K$ is Feller, $\left\{T_{t}\right\}$ is strong Feller (then all semigroups involved are strongly Feller; see $\S 5)$, but $V(x)$ is not continuous, then (2.17) could not hold (in $B$ ) and (2.8) could not be simplified.

(2) Set $K=0$. Then, $\bar{T}_{t}=T_{t}^{\circ}$ and $P^{\prime}$ is the subprocess of $\left\{x_{t}\right\}$ associated with $\left\{\exp \left(-\phi_{t}\right)\right\}$. The uniqueness criterion (2.9) is straightforward and

$$
A_{\lambda}^{\circ}=A_{\lambda}\left(I+S_{\lambda}\right) \text {. }
$$

(Theorem 2.1 requires $S_{\lambda} 1(x)<\infty$, all $x$, but one could argue directly from (4.2) and Theorem 3.1 with no extra hypotheses beyond (2.1).) If $E_{x}(\zeta) \leqq C$, then $a$ posteriori $E_{x}^{\circ}(\zeta) \leqq C$ and $A^{\circ}=A(I+S)$.

These formulas, in the form of operator inclusions, were obtained for the strong generator in [2, Chapter 9] and [21] under assumptions similar to (2.10). Operator equality in (2.18) was obtained for large $\lambda$ by [2], who also remarked that the inclusion (2.18) also held for the weak generator.

A converse of Theorem 2.1, giving conditions implying that a process $P=\left\{x_{t}, B_{t}\right\}$ is a process $P^{\prime}$ for some other process $P$, is given in $\S 9$. In $\$ 5.2$ (with some extra conditions on $\left.\left\{\phi_{t}\right\}\right)$ we derive a formula which is the analogue of the Feller forwards equation for continuous time Markov chains ([1], [3]). We note that if $P(t, x, A)$ $=\delta_{x}(A)$, then (2.5) reduces to the Feller backwards equation for $\bar{P}(t, x, A)$ and (5.11) reduces to the forwards equation (see §5.2).

2.1. We conclude $\S 2$ with some applications of Theorems 2.1-2.4 to partial differential equations. Let

$$
A_{x}=\sum_{1}^{n} \sum_{1}^{n} a_{i j}(x) \partial^{2} / \partial x_{i} \partial x_{j}+\sum_{1}^{n} b_{i}(x) \partial / \partial x_{i}+c(x)
$$

be a differential operator in $R^{n}$, where $\left\{a_{i j}(x)\right\}$ is uniformly elliptic, the coefficients are uniformly Hölder continuous and bounded, and $c(x) \leqq 0$. Let $\mathcal{O}$ be a bounded open set in $R^{n}$ with $C^{3}$-boundary $\partial \mathcal{O}$. We consider first the natural diffusion process $P$ in $\mathcal{O}$ corresponding to the generator (2.19) which terminates in $\partial \mathcal{O}$, and show the effect of jumping induced by a local time on a submanifold of codimension one in the interior of $\mathcal{O}$. Secondly we prove a theorem which is perhaps more usual from a partial differential equations point of view, which results when a diffusion process $P$ with reflecting boundary condition on $\partial \mathcal{O}$ is forced to branch into copies of itself on $\partial \mathcal{O}$. Alternate versions of the two theorems are possible; e.g., with a nonlinear boundary condition on the submanifold in place of (2.21), or a boundary condition of lateral type on $\partial \mathcal{O}$ instead of (2.24).

THEOREM 2.5 (see $\S 8$ ). Let $\Gamma$ be a closed submanifold of $\mathcal{O}$ of class $C^{1+\alpha}$ and codimension one, and choose $\beta(x) \in C(\Gamma)$ with $\beta(x) \geqq 0, \beta(x)=0$ on $\partial \Gamma$. Let $K(x, A)$ be a Feller substochastic kernel on $\mathcal{O}$, and let $\bar{P}$ be the process resulting from Theorem 
2.1 for $\left\{\phi_{t}\right\}$ being local time on $\Gamma$ with weight $\beta(x)$ (see §8) as above. Then, if $f(x) \in C(\overline{\mathcal{O}})$, $u(x, t)=\bar{E}_{x}\left(f\left(x_{t}\right)\right)=\bar{T}_{t} f(x)$ is the unique solution of

$$
\begin{gathered}
u(x, t)=0 \text { on } \partial \mathcal{O} \quad(t>0), \quad u(x, 0)=f(x), \\
\partial / \partial t u(x, t)=A_{x} u(x, t) \text { in } \mathcal{O}-\Gamma, \quad t>0, \\
\frac{1}{2}\left(D_{v}^{+}+D_{v}^{-}\right) u(x, t)=-\beta(x)\left(\int_{\mathcal{O}} u(y, t) K(x, d y)-u(x, t)\right)
\end{gathered}
$$

for all $x \in \Gamma$, where $D_{v}^{ \pm}$are the twin outwards-pointing conormal derivatives at $x \in \Gamma$. Finally, $f(x) \in \mathscr{D}(\bar{A})$ iff $f(x)=g(x)+k(x)$, where $g(x) \in \mathscr{D}(A), k(x) \in C_{0}(\mathcal{O}) \cap C^{2}(\mathcal{O}-\Gamma)$, $A_{x} k(x) \equiv 0$ in $\mathcal{O}-\Gamma$, and $f(x)$ satisfies $(2.21) .\left(C_{0}(\mathcal{O})=\{f \in C(\overline{\mathcal{O}}): f=0\right.$ on $\partial \mathcal{O}\}$.)

RemarKs. (1) Note $D_{v}^{+} g(x)=-D_{v}^{-} g(x)$ if $g \in C^{1}(\mathcal{O})$; thus (2.21) represents a jump condition in the conormal derivative as one crosses $\Gamma$. Also, $\mathscr{D}(A)$ can be characterized in terms of Sobolev spaces [20]; in particular $\mathscr{D}(A)$ is the same as when $A_{x}=\nabla^{2}$, and $\mathscr{D}(A) \subseteq C^{1}(\mathcal{O})$.

(2) If $A_{x}=\frac{1}{2} \nabla^{2}, P$ is Brownian motion terminating on $\partial \mathcal{O}$. Hence $f \in \mathscr{D}(\bar{A})$ iff $f$ $=g+k$, where $g \in \mathscr{D}(A)$ and $k(x)$ is harmonic in $\mathcal{O}-\Gamma$ with $k(x)=0$ on $\partial \mathcal{O}$ and such that (2.21) holds for $f(x)$.

Theorem 2.6 (see §8). Assume $a_{i j}(x) \in C^{2+\alpha}\left(R^{n}\right), b_{i}(x) \in C^{1+\alpha}\left(R^{n}\right)$, and choose functions $\left\{\beta(a), p_{n}(a)\right\}$ on $\partial \mathcal{O}$ such that $\beta(a) \geqq 0, \beta(a) \in C^{\alpha}(\partial \mathcal{O})$ and

$$
\begin{aligned}
& \text { (i) } p_{n}(a) \geqq 0, \quad \sum_{0}^{\infty} p_{n}(a)=1, \\
& \text { (ii) } \sum_{0}^{\infty}\left|p_{n}(a)-p_{n}(b)\right| \leqq C|a-b|^{\alpha} .
\end{aligned}
$$

Let $\bar{P}$ be the branching process on $X=\bigcup \overline{\mathcal{O}}^{n}$ corresponding to $P$ with reflecting boundary condition on $\partial \mathcal{O}$ and generator (2.19), where $\left\{\phi_{t}\right\}$ is local time on $\partial \mathcal{O}$ with weight $\beta(a)$, and such that if $\bar{P}$ branches at $x=a \in \partial \mathcal{O}, n$ descendents are then released at a with probability $p_{n}(a)$. Then if $f(a) \in C(\overline{\mathcal{O}}),|f(a)| \leqq C<1$, and $u(a, t)$ is given by (2.13), we conclude $u(a, t) \in C^{2}(\mathcal{O}) \cap C^{1}(\overline{\mathcal{O}})$ for fixed $t>0$ and $u(a, t)$ is the unique solution of $(t>0)$

$$
\begin{gathered}
\left(\partial / \partial t-A_{a}\right) u(a, t)=0 \quad \text { in } \mathcal{O}, \quad u(a, 0)=f(a) \\
D_{v} u(a, t)=-\beta(a)\left(\sum_{0}^{\infty} p_{n}(a) u(a, t)^{n}-u(a, t)\right)
\end{gathered}
$$

for all $a \in \partial \mathcal{O}$, where $D_{v}$ is the inwards-pointing conormal derivative on $\partial \mathcal{O}$. Moreover, if $|f(a)| \leqq C<1, \hat{f}(x) \in \mathscr{D}(\bar{A})$ iff $f(a)=g(a)+k(a)$, where $g(a) \in \mathscr{D}(A)$ in $\mathcal{O}, k(a) \in$ $C^{2}(\mathcal{O}) \cap C^{1}(\overline{\mathcal{O}}),\left(A_{a}-\lambda\right) k(a)=0$ for some $\lambda>0$, and $f(a)$ satisfies the boundary condition (2.24).

In Theorem 2.6, the process $\bar{P}$ branches on $\partial \mathcal{O}$ into copies of itself at the same 
location in $\partial \mathcal{O}$. More complicated branching rules could, of course, be used and would lead to more complicated versions of (2.24).

REMARKS. (3) The condition that $p_{n}(a) \geqq 0$ in (2.22) is not essential as long as the series $\sum p_{n}(a)$ converges absolutely and uniformly; one can construct "antiworlds" for the branching particles to visit and return and a comparable expectation to solve (2.23), (2.24) with $\left\{p_{n}(a)\right\}$ of varying sign ([14], [26]). Similarly, the term “ $-u(a, t)$ " can be removed under certain circumstances ([14], [15], [26]).

(4) Parabolic linear partial differential equations with nonlinear boundary conditions have been considered by various authors (e.g. $[4, \S 7.5])$ but always under the assumption that the boundary condition corresponding to (2.24) is monotone in $u$. These results, especially in view of the third remark, do not seem comparable. Also, we remark that (2.23), (2.24) was regarded as holding "in a weak sense" by Ikeda, Nagasawa, and Watanabe $([6, \mathrm{II}])$ on the basis of results in [17], but no further discussion or proof of e.E. differentiability was given.

Theorems (2.1)-(2.4) were presented in a colloquium at the Courant Institute in April, 1968.

3. Lemmas on multiplicative functionals. Here we consider the additive functional $\left\{\phi_{t}\right\}$ in multiplicative form. We call a collection of random variables $\left\{\alpha_{t}(\omega)\right\}$ a (contractive) multiplicative functional of the process $P$ if $\alpha_{t}(\omega)=\exp \left(-\phi_{t}(\omega)\right)$, where $\left\{\phi_{t}\right\}$ satisfies (2.1) with (2.1 (iii)) replaced by (iii)' $\phi_{0}=0,0 \leqq \phi_{t} \leqq \infty$. Thus $\alpha_{t}(\omega) \downarrow$ in $t$, and $\alpha_{t+s}=\alpha_{t} \theta_{t} \alpha_{s}$. In the first part of $\S 3$ we also assume that the analogue of (2.1 (iii)) holds, i.e.

$$
\alpha_{t}(\omega)>0 \text { for all } t \text { and } \omega .
$$

For $\tau(\omega)$ as in (2.3), we define operators

$$
\begin{aligned}
& \text { (i) } S_{\lambda}^{\circ} f(x)=E_{x}\left(e^{-\lambda \tau} f\left(x_{\tau}\right)\right)=-E_{x}\left(\int_{0}^{\infty} e^{-\lambda s} f\left(x_{s}\right) d \alpha_{s}\right) \text {, } \\
& \text { (ii) } S_{\lambda} f(x)=-E_{x}\left(\int_{0}^{\infty} e^{-\lambda s} f\left(x_{s}(\omega)\right) d \alpha_{s} / \alpha_{s-}\right)
\end{aligned}
$$

for nonnegative Borel functions $f(x)$. If $\alpha_{t}(\omega)=\exp \left(-\phi_{t}(\omega)\right)$, then (3.2 (ii)) reduces to (2.7). The purpose of this section is to prove (see also Theorem 3.2)

THEOREM 3.1. For Borel functions $f(x), 0 \leqq f(x) \leqq C, \lambda>0$,

$$
\text { (i) } \quad \sum_{1}^{\infty}\left(S_{\lambda}^{\circ}\right)^{n} f(x)=S_{\lambda} f(x) \text {, }
$$$$
\text { (ii) } \lim _{n \rightarrow \infty}\left(S_{\lambda}^{\circ}\right)^{n} f(x)=0 \text { for all } x \text {. }
$$

We begin by extending the probability space on which $P=\left\{x_{t}, B_{t}\right\}$ is defined to construct a sequence of nonnegative random variables $\left\{\tau_{n}\right\}$ such that $\tau_{0}=0$ and

$$
P_{x}\left(\tau_{n+1}>t / B_{\infty}, \tau_{1}, \tau_{2}, \ldots, \tau_{n}\right)=\chi_{\left[\tau_{n}>t\right]}+\chi_{\left[\tau_{n} \leqq t\right]} \theta_{\tau_{n}} \alpha_{t-\tau_{n}^{\circ}}(\omega)
$$

where the superscript ${ }^{\circ}$ here means that the preceding $\theta$-operator is to be ignored 
by the variable superscripted. The variables $\left\{\tau_{n}\right\}$ are the successive "random times" of $\S 1$ in the special case where $K(x, A)$ puts mass one at $x$. By defining

$$
\alpha_{t}^{(n)}=P_{x}\left(\tau_{n}>t / B_{\infty}\right),
$$

we obtain a sequence of (not in general multiplicative) functionals of the process $P$ satisfying the recurrence relation

$$
\alpha_{t}^{(n)}=\alpha_{t}^{(n-1)}-\int_{0}^{t} \theta_{u} \alpha_{t-u} d \alpha_{u}^{(n-1)}, \quad n \geqq 2 .
$$

Equivalently we could have simply defined $\alpha_{t}^{(1)}=\alpha_{t}$ and $\left\{\alpha_{t}^{(n)}\right\}$ by (3.6). In particular, $\alpha_{t}^{(n)}$ is $B_{t}$-measurable for all $t$, is right continuous in $t$ (here any integral $\int_{a}^{b} \phi(u) d f(u)$ means the integral over the cell $(a, b])$, and $0 \leqq \alpha_{t}^{(1)} \leqq \alpha_{t}^{(2)} \leqq \ldots \leqq 1$. The importance of the $\left\{\alpha_{t}^{(n)}\right\}$ for Theorem 3.1 is

Lemma 3.1. For all $f(x) \in \mathscr{L}^{\infty}(X), n \geqq 1$,

$$
\left(S_{\lambda}^{\circ}\right)^{n} f(x)=E_{x}\left(\exp \left(-\lambda \tau_{n}\right) f\left(x_{\tau_{n}}\right)\right)=-E_{x}\left(\int_{0}^{\infty} e^{-\lambda s} f\left(x_{s}\right) d \alpha_{s}^{(n)}\right) .
$$

Proof. By induction on $n$

$$
\begin{aligned}
\left(S_{\lambda}^{\circ}\right)^{n} f(x)=\left(S_{\lambda}^{\circ}\right)^{n-1} S_{\lambda}^{\circ} f(x) & =E_{x}\left(\exp \left(-\lambda \tau_{n-1}\right) S_{\lambda}^{\circ} f\left(x_{\tau_{n-1}}\right)\right) \\
& =-E_{x}\left(\exp \left(-\lambda \tau_{n-1}\right) E_{x_{\tau_{n-1}}}\left(\int_{0}^{\infty} e^{-\lambda s} f\left(x_{s}\right) d \alpha_{s}\right)\right) .
\end{aligned}
$$

We would now like to use one of the several equivalent forms of the strong Markov property for $\tau_{n-1}$. However, we are only given here that $P=\left\{x_{t}, B_{t}\right\}$ is strongly Markov, which guarantees the strong Markov property for stopping times $\beta(\omega)$ with $\{\beta>t\} \in B_{t}$ for all $t$. It does turn out, though, that condition (3.5) with $\alpha_{t}^{(n)}$ being $B_{t}$-measurable for all $t$ is sufficient for the strong Markov property for $\tau_{n}$. We deter a proof to $§ 5.2$. In any event, by the strong Markov property at $\tau_{n-1}$,

$$
\begin{aligned}
\left(S_{\lambda}^{\circ}\right)^{n} f(x) & =-E_{x}\left(\exp \left(-\lambda \tau_{n-1}\right) \theta_{\tau_{n-1}} \int_{0}^{\infty} e^{-\lambda s} f\left(x_{s}\right) d \alpha_{s}\right) \\
& =E_{x}\left(\int_{0}^{\infty} \int_{0}^{\infty} e^{-\lambda(t+s)} f\left(x_{s+t}\right) \theta_{t} d \alpha_{s} d \alpha_{t}^{(n-1)}\right) \\
& =-E_{x}\left(\int_{0}^{\infty} e^{-\lambda u} f\left(x_{u}\right) d \alpha_{u}^{(n)}\right)
\end{aligned}
$$

as a direct result of (3.6) and

LEMMA 3.2. Assume $f(s), g_{t}(s)$ are right-continuous, real-valued functions on $[0, \infty)$ which are decreasing in $s$ and satisfy $f(0)=g_{t}(0)=1,0 \leqq f(s) \leqq 1,0 \leqq g_{t}(s) \leqq 1$. Set

$$
h(t)=f(t)-\int_{0}^{t} g_{u}(t-u) d f(u)
$$


Then for any nonnegative Borel function $\phi(u)$,

$$
\int_{0}^{\infty} \int_{0}^{\infty} \phi(s+t) g_{t}(d s) f(d t)=-\int_{0}^{\infty} \phi(u) d h(u)
$$

Proof. Straightforward if $\phi(u)=\chi_{[0, A]}(u)$; the general result follows by monotone class arguments. The key result for the proof of Theorem 3.1 is

LEMMA 3.3. For jointly measurable functions $g(s, \omega) \geqq 0, t>0$,

$$
\sum_{n=1}^{\infty} \int_{0}^{t} g(s, \omega) d \alpha_{s}^{(n)}=\int_{0}^{t} g(s, \omega) d \alpha_{s} / \alpha_{s-} .
$$

Proof. Since $\alpha_{t}(\omega)>0,(3.6)$ implies

$$
\begin{aligned}
\alpha_{t}^{(n)}= & \alpha_{t}^{(n-1)}-\alpha_{t} \int_{0}^{t} 1 / \alpha_{u} d \alpha_{u}^{(n-1)} \\
= & \alpha_{t}^{(n-1)}-\alpha_{t}\left(\alpha_{t}^{(n-1)} / \alpha_{t}-1\right)+\alpha_{t} \int_{0}^{t} \alpha_{u-}^{(n-1)} d\left(1 / \alpha_{u}\right) ; \\
& \alpha_{t}^{(n)} / \alpha_{t}=1-\int_{0}^{t} \alpha_{u-}^{(n-1)} / \alpha_{u-} d \alpha_{u} / \alpha_{u}
\end{aligned}
$$

by integration by parts. Secondly, the identity $\alpha_{t}^{(n)}=\alpha_{t} \alpha_{t}^{(n)} / \alpha_{t}$ and (3.8) yields

$$
d \alpha_{t}^{(n)}=\alpha_{t} d\left(\alpha_{t}^{(n)} / \alpha_{t}\right)+\alpha_{t-}^{(n)} / \alpha_{t-} d \alpha_{t}=\left(\alpha_{t-}^{(n)}-\alpha_{t-}^{(n-1)}\right) d \alpha_{t} / \alpha_{t-}, \quad n \geqq 2,
$$

where the " $d$ " notation means that the corresponding integrals add in that fashion. Thus, since $\alpha_{t}^{(n-1)} \leqq \alpha_{t}^{(n)}$ and $d \alpha_{t}^{(1)}=\alpha_{t-} d \alpha_{t} / \alpha_{t-}$,

$$
\sum_{1}^{\infty} d \alpha_{t}^{(n)}=h(t, \omega) d \alpha_{t} / \alpha_{t-}
$$

where $h(t, \omega)=\lim _{n \rightarrow \infty} \alpha_{t-}^{(n)}(\omega) \leqq 1$. Hence, to complete the proof of Lemma 3.3 , it only remains to show

$$
\lim _{n \rightarrow \infty} \alpha_{T}^{(n)}(\omega)=1, \quad 0 \leqq T<\infty
$$

Let $t$ be a point of continuity of $h(s, \omega)$; then by Fatou's lemma in (3.8)

$$
\begin{aligned}
h(t, \omega) & \geqq \alpha_{t}+\alpha_{t} \int_{0}^{t} h(u+, \omega) d\left(1 / \alpha_{u}\right) \\
& =\alpha_{t}+\alpha_{t}\left(h(t) / \alpha_{t}-1\right)-\alpha_{t} \int_{0}^{t} d h(u) / \alpha_{u-}, \\
h(t, \omega) & \geqq h(t, \omega)-\alpha_{t} \int_{0}^{t} d h(u) / \alpha_{u-} .
\end{aligned}
$$

However $h(t, \omega) \downarrow$ in $t$; thus $d h(u) \equiv 0$ and $h(t, \omega) \equiv 1$.

Proof of Theorem 3.1. Equation (3.3 (i)) follows by taking $g(s, \omega)=e^{-\lambda s} f\left(x_{s}(\omega)\right)$ in (3.7); for (3.3 (ii)), note that for any $T>0$

$$
\left(S_{\lambda}^{\circ}\right)^{n} f(x)=-E_{x}\left(\int_{0}^{\infty} e^{-\lambda s} f\left(x_{s}\right) d \alpha_{s}^{(n)}\right) \leqq C e^{-\lambda T}+C E_{x}\left(1-\alpha_{T}^{(n)}\right)
$$

and (3.9) implies the rest of Theorem 3.1. 
Corollary 3.1. Assuming $\alpha_{t}(\omega)>0$ for all $t, \omega, \tau_{n}(\omega) \rightarrow \infty$ a.s. $P_{x}$, all $x$.

ProOF. $\tau_{n} \uparrow$ by (3.4), but $P_{x}\left(\tau_{n} \leqq T\right)=E_{x}\left(1-\alpha_{T}^{(n)}\right) \rightarrow 0$.

3.1. Let $\left\{\alpha_{t}(\omega)\right\}$ be a contractive multiplicative functional as before. In this section we consider the limiting behavior of the "successive random times" $\left\{\tau_{n}\right\}$ with, instead of (3.1), the weaker assumption

(i) If $\beta(\omega)=\sup \left\{t: \alpha_{t}(\omega)>0\right\}$, then $P_{x}\left(\beta<\infty, \alpha_{\beta-}=0\right)=P_{x}(\beta<\infty)$.

(ii) For any $t, \theta_{s} \alpha_{t}(\omega)$ is $(s, \omega)$-measurable.

That is, if $\alpha_{t}(\omega)=\exp \left(-\phi_{t}(\omega)\right)$, the additive functional $\left\{\phi_{t}\right\}$ may become infinite as long as it does not suddenly jump to infinity. The analogue of Theorem 3.1 is

TheOREM 3.2. For $\left\{\tau_{n}\right\}$ defined by (3.4), $f(x) \in \mathscr{L}^{\infty}(X), f(x) \geqq 0$,

$$
\begin{gathered}
\tau_{n}(\omega) \rightarrow \beta(\omega) \text { a.s. } P_{x}, \quad \text { all } x, \\
\sum_{1}^{\infty}\left(S_{\lambda}^{\circ}\right)^{n} f(x)=S_{\lambda} f(x) .
\end{gathered}
$$

Moreover, if $\left\{x_{t}(\omega)\right\}$ is quasi-left continuous with left limits $\left\{x_{t-}(\omega)\right\}$ and $f(x) \in B C(X)$

$$
\lim _{n \rightarrow \infty}\left(S_{\lambda}^{\circ}\right)^{n} f(x)=E_{x}\left(e^{-\lambda \beta} f\left(x_{\beta}\right)\right) .
$$

We recall that a Markov process $\left\{x_{t}, B_{t}\right\}$ is quasi-left continuous if

$$
P_{x}\left(x_{\beta_{n}} \rightarrow x_{\beta} \text { a.s., } \beta<\infty\right)=P_{x}(\beta<\infty)
$$

for any sequence of stopping times $\left\{\beta_{n}, \beta\right\}$ with $\beta_{n} \uparrow \beta$. Equation (3.11) is curious since none of the $\left\{\tau_{n}\right\}$ are, in general, $B_{\infty}$-measurable, while $\beta(\omega)$, defined by (3.10), of course is. The proof of Theorem 3.2 depends on

LEMMA 3.4. For $\left\{\alpha_{t}^{(n)}, \beta\right\}$ as before

$$
\begin{array}{ll}
\alpha_{T}^{(n)}(\omega) \rightarrow 1, & 0 \leqq T<\beta(\omega), \\
\alpha_{T}^{(n)}(\omega) \equiv \alpha_{\beta}^{(n)}(\omega)=0, & \beta(\omega) \leqq T<\infty .
\end{array}
$$

Proof. If $0 \leqq T<\beta(\omega)$, then $\alpha_{t}(\omega)>0$ for $0 \leqq t \leqq T$, and $\alpha_{T}^{(n)} \rightarrow 1$ follows as before. Now assume $T_{0}<T<\beta(\omega)$ and by induction $\alpha_{\beta-}^{(n-1)}(\omega)=0$. Then by (3.8)

$$
\begin{aligned}
& \alpha_{T}^{(n)} \leqq \alpha_{T}+\alpha_{T} \int_{0}^{T_{0}} d\left(1 / \alpha_{u}\right)+\alpha_{T} \alpha_{T_{0}}^{(n-1)} \int_{0}^{T} d\left(1 / \alpha_{u}\right), \\
& \alpha_{T}^{(n)} \leqq \alpha_{T} / \alpha_{T_{0}}+\alpha_{T_{0}}^{(n-1)},
\end{aligned}
$$

and $\alpha_{\beta-}^{(n)}=0$. By monotonicity $\alpha_{T}^{(n)} \equiv 0$ for $T \geqq \beta$. Q.E.D.

Proof of Theorem 3.2. First $\tau_{n} \uparrow$ by (3.4), and

$$
P_{x}\left(\beta-\varepsilon<\tau_{n} \leqq \beta\right)=E_{x}\left(\alpha_{\beta}^{(n)}-\alpha_{\beta}^{(n)}\right) \rightarrow 1 .
$$

Hence $\tau_{n} \rightarrow \beta$ a.s. (3.12) follows as before; (3.13) follows from Lemma 3.1 and 
(3.11) if we can prove that $x_{\beta}=x_{\beta}$ a.s. where $\beta<\infty$. However, if

$$
\beta_{n}=\sup \left\{t: \alpha_{t} \geqq 1 / n, t \leqq n\right\},
$$

then $\beta_{n}<\beta, \beta_{n} \uparrow \beta$, and hence $x_{\beta}=x_{\beta}$ a.s. if $\beta<\infty$. Q.E.D.

3.2. If the analogue of (2.2) holds, i.e., if $\alpha_{t}(\omega)$ is continuous in $t$, then (3.6) can be integrated in closed form. I.e., by (3.8)

$$
\alpha_{t}^{(n)} / \alpha_{t}=1+\int_{0}^{t} \alpha_{u}^{(n-1)} / \alpha_{u} d \log 1 / \alpha_{u}
$$

and by induction (see also [7])

$$
\alpha_{t}^{(n)}=\alpha_{t} \sum_{0}^{n-1} 1 / K !\left(\log 1 / \alpha_{t}\right)^{K}
$$

from which Theorem 3.1 is immediate. If

$$
\begin{aligned}
N(t, \omega) & =n, & & \tau_{n}(\omega) \leqq t<\tau_{n+1}(\omega), \\
& =\infty, & & \beta(\omega) \leqq t<\infty,
\end{aligned}
$$

then, given $B_{\infty}, N(t, \omega)$ even becomes a Poisson process with rate $\log 1 / \alpha_{t}$.

\section{Proofs of Theorems 2.1, 2.2, 2.4.}

Proof of Theorem 2.1. Let $\left\{\bar{T}_{t}\right\}$ be a semigroup satisfying (2.5), i.e.,

$$
\bar{T}_{t} f(x)=T_{t}^{\circ} f(x)+E_{x}\left(x_{[\tau \leqq t]} \int_{X} \bar{T}_{t-\tau} f(y) K\left(x_{\tau}, d y\right)\right)
$$

for all $f(x) \in B$. Taking Laplace transforms

$$
\begin{aligned}
\bar{R}_{\lambda} f(x) & =R_{\lambda}^{\circ} f(x)+\int_{0}^{\infty} e^{-\lambda t} E_{x}\left(\chi_{[\tau \leqq t]} \int_{X} \bar{T}_{t-\tau} f(y) K\left(x_{\tau}, d y\right)\right) d t \\
& =R_{\lambda}^{\circ} f(x)+E_{x}\left(\int_{\tau}^{\infty} \int_{X} e^{-\lambda t} \bar{T}_{t-\tau} f(y) K\left(x_{\tau}, d y\right) d t\right) \\
& =R_{\lambda}^{\circ} f(x)+E_{x}\left(e^{-\lambda \tau} \int_{X} \bar{R}_{\lambda} f(y) K\left(x_{\tau}, d y\right)\right) \\
& =R_{\lambda}^{\circ} f(x)+S_{\lambda}^{\circ} K \bar{R}_{\lambda} f(x)
\end{aligned}
$$

for $S_{\lambda}^{\circ}$ defined by (3.2). Hence $R_{\lambda}^{\circ} f(x)=\left(I-S_{\lambda}^{\circ} K\right) \bar{R}_{\lambda} f(x)$. Now, by the strong Markov property at $\tau$ (see Theorem 5.4) $T_{t} f(x)=T_{t}^{\circ} f(x)+E_{x}\left(\chi_{(\tau) t)} T_{t-\tau} f\left(x_{\tau}\right)\right)$ and by the same argument $R_{\lambda}^{\circ} f(x)=\left(I-S_{\lambda}^{\circ}\right) R_{\lambda} f(x)$. Hence for all $N$

$$
\left(\sum_{0}^{N}\left(S_{\lambda}^{\circ}\right)^{n}\right) R_{\lambda}^{\circ} f(x)=\left(I-\left(S_{\lambda}^{\circ}\right)^{N+1}\right) R_{\lambda} f(x)=R_{\lambda} f(x)-\left(S_{\lambda}^{\circ}\right)^{N+1} R_{\lambda} f(x) .
$$

Taking $N \rightarrow \infty$ and applying Theorem 3.1 (since any $f(x) \in B$ is the difference of two nonnegative bounded Borel functions) gives

$$
\begin{aligned}
& R_{\lambda} f(x)=\left(I+S_{\lambda}\right) R_{\lambda}^{\circ} f(x), \\
& R_{\lambda} f(x)=\left(I+S_{\lambda}\right)\left(I-S_{\lambda}^{\circ} K\right) \bar{R}_{\lambda} f(x) .
\end{aligned}
$$


Thus $\left(I+S_{\lambda}\right)\left(I-S_{\lambda}^{\circ} K\right)$ maps $\mathscr{D}(\bar{A})$ onto $\mathscr{D}(A)$ and

$$
A_{\lambda}\left(I+S_{\lambda}\right)\left(I-S_{\lambda}^{\circ} K\right) \bar{R}_{\lambda}=-I, \quad \bar{A}_{\lambda} \subseteq A_{\lambda}\left(I+S_{\lambda}\right)\left(I-S_{\lambda}^{\circ} K\right) .
$$

Next, if $0 \leqq f(x) \leqq C$, again by Theorem 3.1,

$$
S_{\lambda} S_{\lambda}^{\circ} f(x)=S_{\lambda} f(x)-S_{\lambda}^{\circ} f(x)
$$

and as soon as $S_{\lambda}|f|(x)<\infty$ for all $x$ (note $S_{\lambda}|f|(x) \leqq\|f\| S_{\lambda} 1(x)$ )

$$
\left(I+S_{\lambda}\right)\left(I-S_{\lambda}^{\circ} K\right) f=f+S_{\lambda} f-S_{\lambda}^{\circ} K f-S_{\lambda} S_{\lambda}^{\circ} K f=\left(I-S_{\lambda}(K-I)\right) f
$$

and hence by (4.2)

$$
\bar{A}_{\lambda} \subseteq A_{\lambda}\left(I-S_{\lambda}(K-I)\right)
$$

COROLlaRY 4.1. If we remove the condition that $S_{\lambda} 1(x)<\infty$ for all $x$, Theorem 2.1 remains valid with (4.4) or (2.8) replaced by (4.2).

Proof of Theorem 2.2. For $\lambda>0, \bar{A}_{\lambda}$ is one-one and onto $B$, and the expression on the right-hand side of (4.4) preserves $B$ by definition. Hence it is strict extension of $\bar{A}_{\lambda}$ iff there exists some nonzero $f(x) \in B$ such that

$$
A_{\lambda}\left(I-S_{\lambda}(K-I)\right) f(x)=0 .
$$

Since $A_{\lambda}$ is one-one on $\mathscr{D}(A),\left(I-S_{\lambda}(K-I)\right) f=0$. Hence $f(x)=S_{\lambda}(K-I) f(x)$ and by (3.3)

$$
S_{\lambda}^{\circ} f(x)=S_{\lambda}^{\circ} S_{\lambda}(K-I) f(x)=f(x)-S_{\lambda}^{\circ}(K-I) f(x)=f(x)-S_{\lambda}^{\circ} K f(x)+S_{\lambda}^{\circ} f(x)
$$

and $f=S_{\lambda}^{\circ} K f$, which is exactly (2.9). Similarly (2.9) implies (4.5) by (4.3), and hence (note that any bounded measurable solution of (2.9) is automatically in $B_{0}$ ) the inclusion (2.8) or (4.4) is proper (in $B$ ) iff there exist solutions of (2.9) (in $B$ ).

Next, we remark that if $(2.8)$ is attained in $B$, then (2.5) can have only one semigroup solution $\left\{\bar{T}_{t}\right\}$ with $\bar{T}_{t}, \bar{R}_{\lambda}$ preserving $B$, since condition (2.9) is independent of $\left\{\bar{T}_{t}\right\}$ (it only depends on $B$ ) and (2.8) prescribes $\bar{A}_{\lambda}$ exactly. It only remains to show that nontrivial solutions of (2.9) imply multiple semigroup solutions of (2.5) in $B_{0}$.

We now define a sequence of functions $\left\{f_{n}(x)\right\}$ by $f_{0}(x) \equiv 1$ and

$$
f_{n+1}(x)=E_{x}\left(e^{-\lambda \tau} \int_{x} f_{n}(y) K\left(x_{\imath}, d y\right)\right) .
$$

Since $f_{1}(x) \leqq f_{0}(x)=1, f_{n}(x) \downarrow$, and by induction $0 \leqq f_{n}(x) \leqq 1$. Hence $f_{n}(x) \downarrow f(x)$. If (2.9) has a solution $g(x)$ with $|g(x)| \leqq 1$, then $|g(x)| \leqq S_{\lambda}^{\circ} K|g|(x) \leqq f_{1}(x)$ and by induction $|g(x)| \leqq f_{n}(x)$. Hence $|g(x)| \leqq f(x)$; also note that by (4.6), $f(x)$ is a solution of (2.9). Thus (2.9) has nonzero bounded solutions iff $f(x) \not \equiv 0$. Next, if $P^{\prime}=\left\{x_{t}^{\prime}, B_{t}^{\prime}\right\}$ is the process constructed in [5], [6] in the context of $\S 1$, let $\left\{\tau_{n}\right\}$ be the successive random times at which "jumping" occurs and let $\Phi(\omega)$ be a random variable measurable with respect to $\left\{x_{t}^{\prime}\right\}$ and $\left\{\tau_{n}\right\}$. Then, if $E_{\Delta}^{\prime}(|\Phi|)=P_{\Delta}^{\prime}\left(\tau_{1}<\infty\right)=0$,

$$
E_{x}^{\prime}\left(\exp \left(-\lambda \tau_{1}\right) \theta_{\tau_{1}} \Phi\right)=E_{x}\left(\exp \left(-\lambda \tau_{1}\right) \int_{X} E_{y}^{\prime}(\Phi) K\left(x_{\imath}, d y\right)\right)
$$


(See [6, II, §2]; here $\theta_{\tau_{1}} \tau_{n}=\tau_{n+1}-\tau_{1}$.) Hence by induction

$$
f_{n}(x)=E_{x}^{\prime}\left(\exp \left(-\lambda \tau_{n}\right)\right)
$$

and $f(x)=E_{x}^{\prime}\left(\exp \left(-\lambda \tau_{\infty}\right)\right)$ where $\tau_{\infty}=\lim \tau_{n}$. Thus (2.9) has nontrivial solutions in $B_{0}$ iff $P_{x}^{\prime}\left(\tau_{\infty}<\infty\right) \not \equiv 0$. The last part of the argument can also be done without reference to the process $\left\{x_{t}^{\prime}, B_{t}^{\prime}\right\}$, since, in the notation of Moyal,

$$
f(x)=\int_{0}^{\infty} e^{-\lambda t} \sigma_{\infty}(d t, x)
$$

([13, p. 245]). Moyal shows that (2.5) has multiple solutions iff $\sigma_{\infty}(t, x) \not \equiv 0$; indeed, an easy calculation shows that any solution of

$$
\bar{T}_{t} f(x)=T_{t}^{\prime} f(x)+E_{x}^{\prime}\left(\chi_{\left[\tau_{\infty} \leqq t\right]} \int_{x} \bar{T}_{t-\tau_{\infty}} f(y) l(d y)\right)
$$

is also a solution of (2.5) for any probability measure $l(A)$ on $X$.

Finally, according to Theorem 5.1, either of conditions (2.10) imply $\left\|S_{\lambda}^{\circ} f\right\|$ $\leqq \beta_{\lambda}\|f\|, \beta_{\lambda}<1$, for all $\lambda>0$, where $\|f\|=\sup _{x}|f(x)|$. Hence if $f(x)$ is any solution of (2.9) in $B_{0},\|f\|=\left\|S_{\lambda}^{\circ} K f\right\| \leqq \beta_{\lambda}\|f\|<\|f\|$ unless $f(x)=0$, and the proof of Theorem 2.2 is complete.

For Theorem 2.4 we need

LEMMA 4.1. Let $\left\{T_{t}\right\}, B, \mathscr{D}(A)$ be as in the discussion after (2.5), and $E_{x}(\zeta) \leqq C$. Then, $A$ is one-one from $\mathscr{D}(A)$ onto $B$, and $R_{0} A \subseteq A R_{0}=-I$, where

$$
R_{0} f(x)=\int_{0}^{\infty} T_{s} f(x) d s=E_{x}\left(\int_{0}^{\zeta} f\left(x_{s}\right) d s\right)
$$

Proof. Clearly $\left|R_{0} f(x)\right| \leqq\|f\| E_{x}(\zeta) \leqq C\|f\|$, and $R_{0}$ is a bounded linear operator on $X$. The proof of the lemma is standard; see e.g. Theorem 1.7' in [2].

Proof of Theorem 2.4. First, if $g(x) \in \mathscr{D}(\bar{A})$, then $g(x)=\bar{R}_{\lambda} f(x)$ for some $f(x) \in B$, and $f(x)=\lambda g(x)-\bar{A} g(x)$. Hence by (4.1), (4.3),

$$
\lambda R_{\lambda} g(x)-R_{\lambda} \bar{A} g(x)=g(x)-S_{\lambda}(K-I) g(x) .
$$

If $S_{0} K|g|(x)<\infty$ and $S_{0}|g|(x)<\infty$, then $S_{\lambda}(K-I) g(x) \rightarrow S(K-I) g(x)$ as $\lambda \rightarrow 0$, and in the limit

$$
-R_{0} \bar{A} g(x)=g(x)-S(K-I) g(x) .
$$

Hence $\bar{A} \subseteq A(I-S(K-I))$. Second, if $E_{x}(\zeta) \leqq C$, then $\bar{A}$ is also one-one and onto by Lemma 4.1, and (2.15) is a proper inclusion iff there exist $g(x) \in B$,

$$
S(|K g-g|)(x)<\infty,
$$

such that $g(x)=S(K-I) g(x)$. As before we conclude $g=S^{\circ} K g$, where $S^{\circ} g(x)$ $=E_{x}\left(g\left(x_{\tau}\right)\right)$. Similarly, if $P_{x}^{\prime}\left(\zeta^{\prime}<\infty\right) \equiv 1, g=S^{\circ} K g$ has nontrivial bounded solutions iff $P_{x}^{\prime}\left(\tau_{\infty}<\infty\right) \not \equiv 0$, etc.

Finally, (2.10) plus $E_{x}(\zeta) \leqq C$ implies $\left\|S^{\circ} f\right\| \leqq \beta\|f\|, \beta<1$, by Theorem 5.2 and a posteriori $\|S f\| \leqq Q\|f\|, Q=\beta /(1-\beta)$. Uniqueness follows as before. 
5. Random lemmas. Let $\left\{\phi_{t}\right\}, \tau, S_{\lambda}$ be as in $\S 2$ and set $S_{\lambda}^{\circ} f(x)=E_{x}\left(e^{-\lambda \tau} f\left(x_{\tau}\right)\right)$. Then

THEOREM 5.1. The two conditions

(i) $P_{x}\left(\tau \leqq t_{0}\right) \leqq \alpha<1$, some $t_{0}>0$,

(ii) $S_{\lambda}^{\circ} 1(x) \leqq \beta_{\lambda}<1, \quad$ all $\lambda>0$,

are equivalent and are implied by

$$
\sup _{x} E_{x}\left(\phi_{t_{0}}\right) \leqq C<\infty, \text { some } t_{0}>0 .
$$

If $\left\{\phi_{t}\right\}$ is continuous in $t$ (for all $\omega$ ), then (5.1) and (5.2) are equivalent, and are equivalent to $S_{\lambda} 1(x) \leqq C_{\lambda}<\infty$, all $\lambda>0$.

Proof. If (5.1 (i)) holds, $S_{\lambda}^{\circ} 1(x)=E_{x}\left(e^{-\lambda \tau}\right) \leqq \alpha+\exp \left(-\lambda t_{0}\right)(1-\alpha)=\beta_{\lambda}<1$ (note $P_{x}(\zeta \leqq \tau<\infty) \equiv 0$ by (2.3)). Given (5.1 (ii)), then conversely, $P_{x}(\tau \leqq t) \leqq e^{\lambda t} E_{x}\left(e^{-\lambda \tau}\right)$ $\leqq e^{\lambda t} \beta_{\lambda}<1$ for sufficiently small $t$. Given (5.2),

$$
S_{\lambda}^{\circ} 1(x)=-E_{x}\left(\int_{0}^{\infty} e^{-\lambda s} d\left(\exp \left(-\phi_{s}\right)\right)\right)=\lambda \int_{0}^{\infty} e^{-\lambda s} E_{x}\left(1-\exp \left(-\phi_{s}\right)\right) d s .
$$

By concavity of the function $\phi(x)=1-e^{-x}$,

$$
\begin{aligned}
S_{\lambda}^{\circ} 1(x) & \leqq \lambda \int_{0}^{\infty} e^{-\lambda s}\left(1-\exp \left(-E_{x}\left(\phi_{s}\right)\right)\right) d s \\
& \leqq \lambda \int_{0}^{t_{0}} e^{-\lambda s}\left(1-e^{-c}\right) d s+\exp \left(-\lambda t_{0}\right)=\beta_{\lambda}<1 .
\end{aligned}
$$

Finally, (5.1 (ii)) implies $S_{\lambda} 1(x) \leqq C_{\lambda}=\beta_{\lambda} /\left(1-\beta_{\lambda}\right)<\infty$ by Theorem 3.1, while if $\left\{\phi_{t}\right\}$ is continuous $S_{\lambda} 1(x)=E_{x}\left(\int_{0}^{\infty} e^{-\lambda t} d \phi_{t}\right) \geqq e^{-\lambda} E_{x}\left(\phi_{1}\right)$. Q.E.D.

THEOREM 5.2. Assume $\left\{\phi_{t}\right\}$ is continuous (i.e. satisfies (2.1), (2.2)), satisfies either (5.1) or (5.2), and $E_{x}(\zeta) \leqq C$. Then $E_{x}\left(\phi_{\zeta}\right)=E_{x}\left(\phi_{\infty}\right) \leqq C^{\prime}$ and $S^{\circ} 1(x) \leqq \beta<1, S 1(x)$ $\leqq C^{\prime}<\infty$.

Proof. By (5.2) and basic results on continuous additive functionals (see e.g. [2, Chapter 6])

$$
\begin{aligned}
E_{x}\left(\phi_{t}\right) \leqq C_{0}(1+t)=O(t) & (\text { as } t \rightarrow \infty), \\
E_{x}\left(\phi_{t}^{2}\right) \leqq 2 \sup _{y} E_{y}\left(\phi_{t}\right)^{2}=O\left(t^{2}\right) & (\text { as } t \rightarrow \infty) .
\end{aligned}
$$

Now, if $P_{x}(\zeta>t) \leqq C_{t}$ for all $x$ and $t$, then $P_{x}(\zeta>2 t)=P_{x}\left(\zeta>t, \theta_{t}(\zeta>t)\right) \leqq C_{t}^{2}$ and $P_{x}(\zeta>t) \leqq C^{\prime \prime} e^{-2 \alpha t}$ for some $\alpha>0$. Hence

$$
\begin{aligned}
E_{x}\left(\phi_{\zeta}\right) & \leqq \sum_{1}^{\infty} E_{x}\left(\phi_{n+1} \chi_{(n \leqq \zeta \leqq n+1)}\right) \\
& \leqq \sum_{1}^{\infty}\left(E_{x}\left(\phi_{n+1}^{2}\right)\right)^{1 / 2}\left(P_{x}(\zeta \geqq n)\right)^{1 / 2} \\
& \leqq C \sum_{1}^{\infty} n e^{-\alpha n}<\infty
\end{aligned}
$$


Similarly, $E_{x}\left(\exp \left(-\phi_{\zeta}\right)\right) \geqq \exp \left(-E_{x}\left(\phi_{\zeta}\right)\right) \geqq \beta>0$ by convexity and

$$
S^{\circ} 1(x)=P_{x}(\tau<\zeta)=E_{x}\left(1-\exp \left(-\phi_{\zeta}\right)\right) \leqq 1-\beta<1 .
$$

Finally, $S 1(x)=E_{x}\left(\phi_{\infty}\right) \leqq C^{\prime}<\infty$ by Theorem 3.1. Q.E.D.

5.1. We next prove a result which would imply, in particular, that if $\left\{T_{t}\right\}$ were strongly Feller and (5.4) held, then $\left\{T_{t}^{\circ}\right\}$ and $\left\{\bar{T}_{t}\right\}$ would also be strongly Feller. We recall that $\mathscr{L}^{\infty}(X)$ denotes the set of all bounded Borel functions.

THEOREM 5.3. Let $Q$ be a closed linear subspace of $\mathscr{L}^{\infty}(X)$, and suppose that $T_{t}: \mathscr{L}^{\infty}(X) \rightarrow Q$ for all $t>0$. Assume also

$$
P_{x}(\tau \leqq t)=\sigma(1) \text { as } t \rightarrow 0 \text { uniformly in } x .
$$

Then $T_{t}^{\circ}: \mathscr{L}^{\infty}(X) \rightarrow Q$ and $\bar{T}_{t}: \mathscr{L}^{\infty}(X) \rightarrow Q$ for all $t>0$, where $\left\{\bar{T}_{t}\right\}$ is the unique solution of (2.5).

Proof. Uniqueness of $\left\{\bar{T}_{t}\right\}$ follows from Theorem 2.2. Next, if $f(x) \in \mathscr{L}^{\infty}(X)$ and $0<s<t$,

$$
\begin{aligned}
\left\|T_{s} T_{t-s}^{\circ} f-T_{t}^{\circ} f\right\|=\left\|\left(T_{s}-T_{s}^{\circ}\right) T_{t-s}^{\circ} f\right\| & \leqq\|f\| \sup _{x} E_{x}\left(1-\exp \left(-\phi_{s}\right)\right) \\
& \leqq\|f\| \sup _{x} P_{x}(\tau \leqq s)
\end{aligned}
$$

where $\|f\|=\sup _{x}|f(x)|$. Since $Q$ is norm closed and $T_{s} T_{t-s}^{\circ} f \in Q$ for all $s$, we conclude $T_{t}^{\circ} \in Q$ for all $t>0$. With reference to (2.5), we remark

$\left|E_{x}\left(\chi_{[\tau \leqq t]} \int_{X} \bar{T}_{t-\tau} f(y) K\left(x_{\tau}, d y\right)\right)-E_{x}\left(\chi_{[\varepsilon \leqq \tau \leqq t]} \int_{X} \bar{T}_{t-\tau} f(y) K\left(x_{\tau}, d y\right)\right)\right| \leqq\|f\| P_{x}(\tau \leqq \varepsilon)$

and

$$
\begin{aligned}
E_{x}\left(\chi_{[\varepsilon \leqq \imath \leqq t]} \int_{X} \bar{T}_{t-\tau} f(y) K\left(x_{\imath}, d y\right)\right) \\
=-E_{x}\left(\int_{\varepsilon}^{t} K \bar{T}_{t-s} f\left(x_{s}\right) d\left(\exp \left(-\phi_{s}\right)\right)\right) \\
=-E_{x}\left(\exp \left(-\phi_{\varepsilon}\right) \theta_{\varepsilon}\left(\int_{0}^{t-\varepsilon} K \bar{T}_{t-\varepsilon-s} f\left(x_{s}\right) d\left(\exp \left(-\phi_{s}\right)\right)\right)\right) \\
=T_{\varepsilon}^{\circ}\left(\bar{T}_{t-\varepsilon}-T_{t-\varepsilon}^{\circ}\right) f(x) \in Q
\end{aligned}
$$

since $T_{t}^{\circ}: \mathscr{L}^{\infty}(X) \rightarrow Q$. Thus both terms on the right-hand side of the basic renewal equation (2.5) belong to $Q$, and $\bar{T}_{t} f(x) \in Q$ as well.

REMARK. If $X$ is compact and $Q=C(X)$, then (5.4) is actually necessary and sufficient. Indeed, if $1-T_{t}^{\circ} 1(x)=P_{x}(\tau \leqq t) \in C(X)$ for all $t>0$, then (5.4) follows by Dini's Theorem.

5.2. An extended strong Markov property This section is devoted to the necessary question of whether the strong Markov property is valid for the random time $\tau$ of (2.3) (and the times $\tau_{n}$ of $\S 3$ ). We are given that $\left\{x_{t}, B_{t}\right\}$ is a strong Markov 
process, which implies the strong Markov property only for random variables $\beta(\omega)$ satisfying $\{\beta>t\} \in B_{t}$ for all $t$. If $\left\{x_{t}\right\}$ were a Feller process with right-continuous paths, or indeed if $\left\{x_{t}\right\}$ merely has right-continuous paths, since $\left\{x_{t}\right\}$ is always Feller in its own fine topology, there is no trouble; the usual Dynkin-Yushkevich proof would go through on the basis of (2.4) or (3.5). In any event, fine topological arguments can be avoided, and we would like to give here a short proof of the strong Markov property for $\tau$ which does not even require that $\left\{x_{t}\right\}$ has right-continuous paths, provided it is progressively measurable and has a metric state space.

THEOREM 5.4. Let $\left\{x_{t}, \mathscr{B}_{t}\right\}$, where $\mathscr{B}_{t}=\bigcap_{[\varepsilon>0]} \mathscr{B}\left[\left\{x_{s}: s \leqq t+\varepsilon\right\}\right], \mathscr{B}_{\infty}=\bigcup \mathscr{B}_{t}$, be a strong Markov process defined on the probability space $\left(\Omega, \mathscr{F}, P_{x}\right)$. Let $\tau(\omega)$ be a nonnegative random variable on $\Omega$ such that

$$
P_{x}\left(\tau>t / \mathscr{B}_{\infty}\right)=\gamma_{t}(\omega) \text { is (ess.) } \mathscr{B}_{t} \text {-meas. }
$$

for all $t$, and define

$$
\mathscr{F}_{\tau}=\left\{E \in \mathscr{F}: P_{x}\left(E, \tau \leqq t / \mathscr{B}_{\infty}\right) \text { is (ess.) } \mathscr{B}_{t} \text {-meas., all } t, x\right\} .
$$

Then, given any $t>0$ and Borel set $A \subseteq X$

$$
P_{x}\left(x_{t+\tau} \in A / \mathscr{F}_{\tau}\right)=P\left(t, x_{\tau}, A\right) \text { a.s. }
$$

where we define $x_{\tau}=\Delta$ if $\tau=\infty$. Other forms of the strong Markov property follow by standard arguments.

Proof. First we remark that $x_{\tau}$ is $\mathscr{F}_{\tau}$-measurable, since

$$
P_{x}\left(x_{\tau} \in A, \tau \leqq t / \mathscr{B}_{\infty}\right)=-\int_{0}^{t} \chi_{A}\left(x_{u}\right) d \gamma_{u}
$$

is $\mathscr{B}_{t}$-measurable by (5.5). Next, we show that for a general $\mathscr{F}_{\tau}$-measurable random variable $\Phi(\omega)$, with $0 \leqq \Phi(\omega) \leqq 1$,

$$
E_{x}\left(\Phi_{\chi_{A}}\left(x_{t+\tau}\right)\right)=E_{x}\left(\Phi P\left(t, x_{\tau}, A\right)\right) .
$$

Let $q_{t}(\omega)$ be a right-continuous increasing $\mathscr{B}_{t}$-measurable version of

$$
q_{t}(\omega)=E_{x}\left(\Phi(\omega) \chi_{[\tau \leqq t]} / \mathscr{B}_{\infty}\right)(\omega)
$$

and let $m$ be a uniformly distributed random variable in $[0,1]$ which is independent of $\left\{x_{t}\right\}$. Define

$$
\beta(\omega)=\sup \left\{t: q_{t}(\omega) \leqq m\right\}, \quad \beta_{s}(\omega)=\sup \left\{t: q_{t}(\omega) \leqq s\right\}, \quad 0 \leqq s \leqq 1 .
$$

Then, $\beta_{s}(\omega)$ are $\mathscr{B}_{\infty}$-measurable stopping times for which the strong Markov property is valid, and

$$
P_{x}\left(\beta \leqq t / \mathscr{B}_{\infty}\right)=P_{x}\left(q_{t}>m / \mathscr{B}_{\infty}\right)=q_{t}(\omega) \text { a.s. }
$$


Hence by repeated use of monotone class arguments (note $x_{\infty}=\Delta$ ) and (5.7)

$$
\begin{aligned}
E_{x}\left(\Phi_{\chi_{A}}\left(x_{t+\tau}\right)\right) & =E_{x}\left(\int_{0}^{\infty} \chi_{A}\left(x_{t+u}\right) d q_{u}\right) \\
& =E_{x}\left(\chi_{A}\left(x_{t+\beta}\right)\right)=E_{x}\left(E_{x}\left(\chi_{A}\left(x_{t+\beta}\right) / m\right)\right) \\
& =\int_{0}^{1} E_{x}\left(\chi_{A}\left(x_{t+\beta_{s}}\right)\right) d s=\int_{0}^{1} E_{x}\left(P\left(t, x_{\beta_{s}}, A\right)\right) d s \\
& =E_{x}\left(P\left(t, x_{\beta}, A\right)\right)=E_{x}\left(\Phi P\left(t, x_{\tau}, A\right)\right)
\end{aligned}
$$

by (5.7) and the strong Markov property for $\beta_{s}$. Q.E.D.

REMARK. The above proof shows, in particular, that the strong Markov property for the process $\left\{x_{t}, \mathscr{B}_{t}\right\}$ is a consequence of the apparently weaker condition

$$
E_{x}\left(\chi_{A}\left(x_{t+\tau}\right)\right)=E_{x}\left(P\left(t, x_{\tau}, A\right)\right)
$$

for all Markov times $\tau$.

5.3. In this section we assume

(i) $\left\{\phi_{t}\right\}$ satisfies (2.1), (2.2), and $S_{\lambda} \chi_{A_{n}}(x)<\infty$ for all $x$ and $n$, where $\left\{A_{n}\right\}$ are Borel sets with $A_{n} \uparrow X$.

(ii) $P(t, x, A)$ has a density $p(t, x, y)$ with respect to a measure " $d y$ " on $X$.

(iii) There exists a nonnegative Borel measure $\mu$ on $X$ such that

$$
\begin{aligned}
E_{x}\left(\phi_{t}\right) & =\int_{0}^{t} \int_{X} p(s, x, y) \mu(d y) d s, \\
E_{x}\left(\int_{0}^{t} f\left(x_{s}\right) d \phi_{s}\right) & =\int_{0}^{t} \int_{X} p(s, x, y) f(y) \mu(d y) d s
\end{aligned}
$$

for all $f(x) \in \mathscr{L}^{\infty}(X)$ with $f(x) \geqq 0$, whenever either side of an equation is finite. (In particular, if $f(x) \leqq \chi_{A_{n}}(x)$ for some $n$.) For Brownian motion, any additive functional satisfying (2.1), (2.2) must be of the above form, and the $\mu$ 's which occur can be explicitly characterized (see [2, Chapter 8], [9]). For example, $\left\{\phi_{t}\right\}$ of Kac type corresponds to $\mu$ of the form $\mu(d y)=V(y) d y$, and "local times" to $\mu$ concentrated on manifolds of codimension one.

Since $P^{\circ}(t, x, A) \leqq P(t, x, A), P^{\circ}(t, x, A)$ also has a density $p^{\circ}(t, x, y)$. Indeed

LEMMA 5.1. For all Borel functions $f(s, x)$

$$
E_{x}\left(\int_{0}^{t} f\left(s, x_{s}(\omega)\right) \exp \left(-\phi_{s}\right) d \phi_{s}\right)=\int_{0}^{t} \int_{x} p^{\circ}(s, x, y) f(s, y) \mu(d y) d s .
$$

Proof. By Laplace transform arguments, it is sufficient to prove

$$
S_{\lambda}^{\circ} f(x)=E_{x}\left(\int_{0}^{\infty} e^{-\lambda s} f\left(x_{s}(\omega)\right) \exp \left(-\phi_{s}\right) d \phi_{s}\right)=\int_{0}^{\infty} \int_{x} e^{-\lambda s} p^{\circ}(s, x, y) f(y) \mu(d y) d s
$$

for $f(x) \geqq 0, S_{\lambda} f(x)<\infty$ (all $x$ ). Denote the double integral above by $J$. Then, writing

$$
T_{t} f(x)=T_{t}^{\circ} f(x)+E_{x}\left(\chi_{(\tau \leqq t)} T_{t-\tau} f\left(x_{\tau}\right)\right)
$$


in terms of densities and using (5.8), (2.7), we obtain

$$
\begin{gathered}
\int_{0}^{\infty} e^{-\lambda t} p(t, x, y) d t=\int_{0}^{\infty} e^{-\lambda t} p^{\circ}(t, x, y) d t+E_{x}\left(\int_{\tau}^{\infty} e^{-\lambda t} p\left(t-\tau, x_{\tau}, y\right) d t\right), \\
S_{\lambda} f(x)=J+E_{x}\left(e^{-\lambda \tau} S_{\lambda} f\left(x_{\tau}\right)\right), \quad S_{\lambda} f(x)=J+S_{\lambda}^{\circ} S_{\lambda} f(x) .
\end{gathered}
$$

By Theorem 3.1, $S_{\lambda}^{\circ} S_{\lambda} f(x)=S_{\lambda} f(x)-S_{\lambda}^{\circ} f(x)$. Hence $S_{\lambda} f(x)=J+S_{\lambda} f(x)-S_{\lambda}^{\circ} f(x)$ and $S_{\lambda}^{\circ} f(x)=J$. Q.E.D.

Equation (2.5) always has a minimal transition function solution, which is obtained by iteration in (2.5). In particular (by iteration) it has a transition density with respect to $d y$. In general, if $\left\{\bar{T}_{t}\right\}$ is any solution of $(2.5)$ with a transition density $\bar{p}(t, x, y)$, then by Lemma 5.1 and (2.5)

$$
\bar{p}(t, x, y)=p^{\circ}(t, x, y)+\int_{0}^{t} \int_{X} p^{\circ}(s, x, z) \int_{X} \bar{p}(t-s, a, y) K(z, d a) \mu(d z) d s .
$$

If $P(t, x,\{x\})=1$ and $\phi_{t}(\omega) \equiv q\left(x_{0}(\omega)\right) t$, this is exactly the Feller backwards equation. For the minimal solution we also have the analogue of the forwards equation.

THEOREM 5.5. Let $\left\{\bar{T}_{t}\right\}$ be the minimal semigroup solution of $(2.5)$ and let $\bar{p}(t, x, y)$ be its transition density (where $\left\{\phi_{t}\right\}$ satisfies (5.8)). Then, given any $f(x) \in \mathscr{L}^{\infty}(X)$ and $x, y \in X$,

$$
\begin{aligned}
\bar{T}_{t} f(x) & =T_{t}^{\circ} f(x)+\int_{0}^{t} \int_{X} \bar{p}(s, x, y) K T_{t-s}^{\circ} f(y) \mu(d y) d s \\
\bar{p}(t, x, y) & =p^{\circ}(t, x, y)+\int_{0}^{t} \int_{X} \bar{p}(s, x, z) \int_{X} p^{\circ}(t-s, a, y) K(z, d a) \mu(d z) d s .
\end{aligned}
$$

Proof. Clearly the two equations are the same. Integration and Lemma 5.1 applied to (5.10) yields $\bar{S}_{\lambda} f(x)=S_{\lambda}^{\circ} f(x)+S_{\lambda}^{\circ} K \bar{S}_{\lambda} f(x)$ where

$$
\bar{S}_{\lambda} f(x)=\int_{0}^{\infty} \int_{X} e^{-\lambda s} \bar{p}(s, x, y) f(y) \mu(d y) d s .
$$

Similarly, the Laplace transform version of (2.5) (see $\S 4$ ) is

$$
\bar{R}_{\lambda} f(x)=R_{\lambda}^{\circ} f(x)+S_{\lambda}^{\circ} K \bar{R}_{\lambda} f(x) .
$$

Now the minimal solution is the result of iteration in (2.5), and hence $\bar{p}(t, x, y)$ is the result of iteration in (5.10). Thus the two equations above are also solved by iteration, and if $f(x) \geqq 0$

$$
\begin{aligned}
\bar{S}_{\lambda} K f(x) & =\sum_{1}^{\infty}\left(S_{\lambda}^{\circ} K\right)^{n} f(x), \\
\bar{R}_{\lambda} f(x) & =R_{\lambda}^{\circ} f(x)+\sum_{1}^{\infty}\left(S_{\lambda}^{\circ} K\right)^{n} R_{\lambda}^{\circ} f(x)=R_{\lambda}^{\circ} f(x)+\bar{S}_{\lambda} K R_{\lambda}^{\circ} f(x)
\end{aligned}
$$

which is exactly (5.11) in Laplace transformation form.

6. Branching Markov processes. Let $P=\left\{x_{t}, B_{t}\right\}$ be a strong Markov process with right-continuous paths in the metric state space $D$, and let $P(t, a, E)$ be its transition function. Set $X=\cup_{0}^{\infty} D^{n}=\{\partial\} \cup D \cup D \times D \cup \cdots$, where $D^{n}$ is the 
usual $n$-fold Cartesian product and $\partial$ is an extra point. The purpose here is to extend the process $P$ (on $D$ ) to a "branching Markov process" (see [5], [6], [19]) $\bar{P}$ on $X$ of the sort discussed in $\S 2$. Our procedure will be similar to that in [5], [6] except for a simpler but less canonical construction; it is given mainly for completeness and later reference. We first extend the process $P$ to a process (which we will also call $P$ ) on $X$ which corresponds to $n$ copies of $P(0 \leqq n<\infty)$ developing independently. If the process $P$ on $D$ is defined on the probability space $\left(\Omega, \mathscr{F}, P_{a}\right)$, the probability space of $P$ on $X$ will be taken to be

$$
\Omega_{X}=\bigcup_{0}^{\infty} \Omega^{n}=\left\{w_{\partial}\right\} \cup \Omega \cup \Omega \times \Omega \cup \ldots
$$

where if $w=\left(\omega_{1}, \omega_{2}, \ldots, \omega_{n}\right) \in \Omega^{n}$ and $0 \leqq t<\infty$,

$$
z_{t}(w)=\left(x_{t}\left(\omega_{1}\right), x_{t}\left(\omega_{2}\right), \ldots, x_{t}\left(\omega_{n}\right)\right) \in D^{n}, \quad z_{t}\left(w_{\partial}\right)=\partial .
$$

The new process $P=\left\{z_{t}, B_{t}^{X}\right\}$ on $X$ will be subject to the transition function $P\left(t, x, E_{1} \times \cdots \times E_{n}\right)=P\left(t, a_{1}, E_{1}\right) P\left(t, a_{2}, E_{2}\right) \cdots P\left(t, a_{n}, E_{n}\right)$ and $P\left(t, x, X-D^{n}\right)=0$ (if $x \in D^{n}$ ) and $P(t, \partial,\{\partial\})=1$, which, as is easily checked, is a transition function on $X$. Let $\left\{\phi_{t}(\omega)\right\}$ be a functional of $P$ on $D$ satisfying (2.1), and define an additive functional $\left\{\psi_{t}\right\}$ of $P$ on $X$ by

$$
\psi_{t}(w)=\phi_{t}\left(\omega_{1}\right)+\phi_{t}\left(\omega_{2}\right)+\cdots+\phi_{t}\left(\omega_{n}\right), \quad \psi_{t}\left(w_{\partial}\right)=0,
$$

where $w=\left(\omega_{1}, \omega_{2}, \ldots, \omega_{n}\right)$. Let $m_{1}, m_{2}, \ldots, m_{n}$ be $n$ random variables independent of $P$ and of one another with $P\left(m_{K}>t\right)=e^{-t}$, and set

$$
\tau_{K}(w)=\sup \left\{t: \phi_{t}\left(\omega_{K}\right) \leqq m_{K}\right\}, \quad \beta(w)=\min _{1 \leqq K \leqq n} \tau_{K}(w)
$$

for $w \in \Omega^{n}$. Then, if $x \in D^{n}$,

$$
\begin{aligned}
P_{x}\left(\beta>t / B_{\infty}^{X}\right) & =P_{x}\left(\phi_{t}\left(w_{1}\right) \leqq m_{1}, \ldots, \phi_{t}\left(w_{n}\right) \leqq m_{n} / B_{\infty}^{X}\right) \\
& =\exp \left(-\phi_{t}\left(\omega_{1}\right)\right) \exp \left(-\phi_{t}\left(\omega_{2}\right)\right) \cdots \exp \left(-\phi_{t}\left(\omega_{n}\right)\right)=\exp \left(-\psi_{t}(w)\right) .
\end{aligned}
$$

Since this is exactly (2.4) with $\left\{\psi_{t}\right\}$ in place of $\left\{\phi_{t}\right\}, \beta(w)$ is the corresponding jumping time. The branching distribution in $X$ will describe the result of one particle being transformed into many particles at multiple locations, the other particles remaining fixed, and is constructed as follows. Assume

(i) $\pi_{0}(a) \in \mathscr{L}^{\infty}(D), 0 \leqq \pi_{0}(a) \leqq 1$.

(ii) For each $E \subseteq X, \pi_{n}(a, E) \in \mathscr{L}^{\infty}(D)$ and $0 \leqq \pi_{n}(a, E) \leqq 1$.

(iii) For each $a \in D, \pi_{n}(a, E)$ is a Borel measure on $D^{n}$.

(iv) $\pi_{0}(a)+\sum_{1}^{\infty} \pi_{n}\left(a, D^{n}\right) \equiv 1$.

For $g(x) \in \mathscr{L}^{\infty}(X)$ and $x=\left(a_{1}, a_{2}, \ldots, a_{n}\right)$, define stochastic kernels $\left\{K_{i}(x, E)\right\}$ by

$$
\begin{aligned}
K_{i} g(x) & =\int_{X} g(y) K_{i}(x, d y) \\
& =g\left(a_{1}, a_{2}, \ldots, a_{i-1}, a_{i+1}, \ldots, a_{n}\right) \pi_{0}\left(a_{i}\right) \\
& +\sum_{1}^{\infty} \int_{D^{m}} g\left(a_{1}, \ldots, a_{i-1}, b_{1}, b_{2}, \ldots, b_{m}, a_{i+1}, \ldots, a_{n}\right) \pi_{m}\left(a_{i}, d b_{1} x \cdots d b_{m}\right) .
\end{aligned}
$$


If $n=1$, the coefficient of $\pi_{0}(a)$ is taken to be $g(\partial)$, and $K_{i} g(\partial)=g(\partial)$. Finally, the branching distribution in $X$ at the branching time $\beta(w)$ is given by

$$
\mu(w, E)=\sum_{i=1}^{n} \chi_{\left[\beta=\tau_{i}\right]} K_{i}\left(z_{\beta}(w), E\right), \quad \mu\left(w_{\partial},\{\partial\}\right)=1 .
$$

The key complication here is that the branching distribution is not a function of the position $z_{\beta}(w)$ of the particles; probabilistically this represents the fact that the particle which caused the branching $\left(\beta=\tau_{K}\right)$ splits into $n$ new particles $(0 \leqq n<\infty)$, the other particles being undisturbed. We are implicitly assuming here that $P_{x}\left(\tau_{i}=\tau_{j}\right)=0$ if $i \neq j$, which is guaranteed by $E_{a}\left(\exp \left(-\phi_{t}\right)\right)$ being continuous in $t$ for all $a$ (e.g. if $\left.E_{a}\left(\phi_{t}\right) \leqq C_{t} \downarrow 0\right)$.

Let $P^{\circ}(t, x, A)=E_{x}\left(\chi_{A}\left(z_{t}\right) \exp \left(-\psi_{t}\right)\right)$, where $P=\left\{z_{t}\right\}$ on $X$. Then, according to Moyal [13], there exists a transition function $\bar{P}(t, x, A)$ on $X$ which is the minimal solution of the equation

$$
\bar{P}(t, x, A)=P^{\circ}(t, x, A)+E_{x}\left[\chi_{[\beta \leqq t]} \int_{X} \bar{P}(t-\beta, y, A) \mu(w, d y)\right]
$$

on $X$. Now $\mu(w, A)$, as defined in (6.3), is an "instantaneous distribution" in the sense of [6]. Hence the path-stitching technique of [6] is also applicable and guarantees a process $\bar{P}=\left\{z_{t}, N_{t}\right\}$ which is a right-continuous strong Markov process in $X$. A partial expression of the fact that individual particles develop independently under $\bar{P}$ is

LeMma 6.1. Let $\beta_{n}$ be the nth branching time (as in $\$ 1$ ) of $\bar{P}$ and let $A_{1}, A_{2}, \ldots, A_{l}$ be Borel sets in $D$. Then, if $x=\left(a_{1}, a_{2}, \ldots, a_{n}\right)$

$$
\begin{aligned}
\bar{P}_{x}\left(z_{t} \in A_{1} \times A_{2} \times \cdots \times A_{l}, \beta_{m} \leqq t<\beta_{m+1}\right) & \\
= & \sum_{\substack{K_{1}+K_{2}+\cdots+K_{n}=m \\
l_{1}+l_{2}+\cdots+l_{n}=l}} \prod_{i=1}^{n} \bar{P}_{a_{i}}\left(z_{t} \in A^{i}, \beta_{K_{i}} \leqq t<\beta_{K_{i}+1}\right)
\end{aligned}
$$

where $A^{i}=A_{p_{i}+1} \times A_{p_{i}+2} \times \cdots \times A_{p_{i+1}}, p_{i}=l_{1}+l_{2}+\cdots+l_{i-1}$, and $A^{i}=\{\partial\}$ if $l_{i}=0$.

Proof. For $1 \leqq i \leqq n$, let $z_{t}^{(i)}(w)$ be the $l_{i}$-tuple of exactly those particles at time $t$ which are descendents of the $i$ th particle (this will be a consecutive batch of components in $\left.z_{t}(w)\right)$, and let $\beta_{l}^{(i)}(w)$ be the $l$ th branching time only among these particles and their predecessors. These quantities are definable in our model, and indeed

$$
\chi_{\left[\beta=\tau_{i}\right]} \theta_{\beta} \beta_{l}^{(i)}=\chi_{\left[\beta=\tau_{i}\right]}\left(\beta_{l+1}^{(i)}-\beta\right), \quad \chi_{\left[\beta=\tau_{i}\right]} \theta_{\beta} \beta_{l}^{(j)}=\chi_{\left[\beta=\tau_{i}\right]}\left(\beta_{l}^{(j)}-\beta\right)
$$

for $j \neq i$. I claim

$$
\bar{P}_{x}\left(z_{t}^{(i)} \in A^{i}, \beta_{K_{i}}^{(i)} \leqq t<\beta_{K_{i}+1}^{(i)}, \text { all } i\right)=\prod_{i=1}^{n} \bar{P}_{a_{i}}\left(z_{t} \in A^{i}, \beta_{K_{i}} \leqq t<\beta_{K_{i}+1}\right)
$$

Given (6.6), the lemma follows by summation. (At the cost of extra computation, one could also argue directly from (6.5).) 
First, by the independence of components of the process $P$ in $X,(6.6)$ is trivial if $m=\sum_{1}^{n} K_{i}=0$ (for any $n$ ). Hence we assume (6.6) by induction for all $n$ and $\sum_{1}^{n} K_{i}^{\prime}=m^{\prime}<m$. Also, by the strong Markov property (i.e. (4.7)) or similar identities in [13]

$$
\begin{gathered}
E_{a}\left(\phi(\tau) \int_{x} \bar{P}_{y}(E) K\left(x_{\tau}, d y\right)\right)=\bar{E}_{a}\left(\phi(\beta) \theta_{\beta} E\right), \\
E_{a}\left(\chi_{[\tau>u]} \bar{P}_{x_{u}}\left(z_{t-u} \in A, \beta_{l} \leqq t-u<\beta_{l+1}\right)\right)=\bar{P}_{a}\left(\tau>u, z_{t} \in A, \beta_{l} \leqq t<\beta_{l+1}\right),
\end{gathered}
$$

where $K(a, E)$ is the kernel defined by (6.2) for $n=1$.

Let $\Omega_{j}(t)=\left\{z_{t} \in A^{j}, \beta_{K_{j}} \leqq t<\beta_{K_{j}+1}\right\}, 1 \leqq j \leqq n$. Then, with the convention that a variable superscripted ${ }^{\circ}$ is held constant in an inner integration, we obtain from the left-hand side of (6.6)

$$
\begin{aligned}
\sum_{i=1}^{n} \bar{P}_{x}(\beta & \left.=\tau_{i} \leqq t, z_{t}^{(j)} \in A^{j}, \beta_{K_{j}}^{(j)} \leqq t<\beta_{K_{j}+1}^{(j)}, \text { all } j\right) \\
& =\sum_{i=1}^{n} \bar{E}_{x}\left(\beta=\tau_{i} \leqq t, \bar{P}_{z_{\beta}}\left(z_{t-\beta^{\circ}}^{(j)} \in A^{j}, \beta_{K_{j}}^{(j)} \leqq t-\beta^{\circ}<\beta_{K_{j}+1}^{(j)}(j \neq i) ;\right.\right. \\
& =\sum_{i=1}^{n} E_{x}\left(\beta=\tau_{i} \leqq t, \prod_{j \neq i}^{n} \bar{P}_{\left(z_{z_{i}}\right)_{j}}\left(\Omega_{j}\left(t-\tau_{i}^{\circ}\right)\right)\right. \\
& \left.\left.=\sum_{i=1}^{i} \beta_{K_{i}-1}^{(i)} \leqq t-\beta^{\circ}<\beta_{K_{i}}^{(i)}\right)\right) \\
& \sum_{x}\left(\tau \leqq t, \prod_{j \neq i}^{n} E_{a_{j}}\left(\tau>\tau_{t-\tau_{i}^{\circ}} \in A^{i}, \beta_{K_{i}-1} \leqq t-\tau_{i}^{\circ}<\beta_{K_{i}}\right) K\left(\left(z_{\tau_{i}}\right)_{i}, d y\right)\right) \\
& \left.\left.=\sum_{i=1}^{n} \bar{E}_{a_{i}}\left(\beta \leqq t-\tau^{\circ}\right)\right)\right)
\end{aligned}
$$

by induction and (6.6) twice, (6.3) and (6.7), where $\Omega_{j}=\Omega_{j}(t)$.

Now, if $K_{j}=0$ and $\beta^{\circ} \leqq t, \bar{P}_{a_{j}}\left(\beta>\beta^{\circ}, \Omega_{j}\right)=\bar{P}_{a_{j}}\left(\Omega_{j}\right)$. Since this factors out of the last expression, we can assume $K_{j}>0,1 \leqq j \leqq n$, in verifying (6.6). But then $\bar{P}_{a_{j}}\left(\Omega_{j}\right)$ $=\bar{P}_{a j}\left(\Omega_{j}, \beta \leqq t\right)$ and, if $\bar{P}_{a}(E \mid \beta=s)$ is the Radon-Nikodym derivative of $\bar{P}_{a}(E, \beta \leqq s)$ with respect to $\bar{P}_{a}(\beta \leqq s)$, we obtain from the left-hand side of (6.6)

$$
\begin{aligned}
\sum_{i=1}^{n} \iint_{0 \leqq s_{i} \leqq s_{j}(\text { all }} \ldots \int_{j \neq i)<t} & \prod_{j=1} \bar{P}_{a_{j}}\left(\Omega_{j} \mid \beta=s_{j}\right) \prod_{j=1}^{n} \bar{P}_{a_{j}}\left(\beta \in d s_{j}\right) \\
& =\int_{0}^{t} \int_{0}^{t} \cdots \int_{0}^{t} \prod_{j=1}^{n} \bar{P}_{a_{j}}\left(\Omega_{j} \mid \beta=s_{j}\right) \prod_{j=1}^{n} \bar{P}_{a_{j}}\left(\beta \in d s_{j}\right) \\
& =\prod_{j=1}^{n} \bar{P}_{a_{j}}\left(\Omega_{j}, \beta \leqq t\right)=\prod_{j=1} \bar{P}_{a_{j}}\left(\Omega_{j}\right)
\end{aligned}
$$

since $\bar{P}_{a}(\beta=s) \equiv 0$, and (6.6) follows for $m$. Q.E.D. 
For a more detailed analysis of the "descendence-structure" of $\left\{z_{t}\right\}$ with $A=0$, see [25].

For an arbitrary $f(a) \in \mathscr{L}^{\infty}(D),|f(a)| \leqq 1$, define a function $\hat{f}(x)$ on $X$ by

$$
\hat{f}(x)=f\left(a_{1}\right) f\left(a_{2}\right) \cdots f\left(a_{n}\right), \quad x=\left(a_{1}, a_{2}, \ldots, a_{n}\right), \quad \hat{f}(\partial)=1 .
$$

Then, an important application of Lemma 6.1 is the branching property ([5], [6])

$$
\bar{T}_{t} \hat{f}(x)=\bar{T}_{t} \hat{f}\left(a_{1}\right) \bar{T}_{t} \hat{f}\left(a_{2}\right) \cdots \bar{T}_{t} \hat{f}\left(a_{n}\right), \quad \bar{T}_{t} \hat{f}(\partial)=1,
$$

which follows from (6.5) by summation and integration. Another application of Lemma 6.1 is a probabilistic interpretation of iteration in the so-called " $S$-equation" for branching processes; see [16].

7. Branching Markov processes, continued. We are particularly interested in those $f(a) \in \mathscr{L}^{\infty}(D)$ for which $\hat{f}(x) \in \mathscr{D}(\bar{A})$ on $X$, since these will allow us to handle certain kinds of nonlinearity on $D$. Let $A$ and $\bar{A}$ be the weak infinitesimal generators (with maximal domain; i.e., $B=B_{0}$ in (2.6)) of $P(t, a, E)$ on $D$ and $\bar{P}(t, x, E)$ on $X$ respectively, and let $S_{\lambda}$ and $S_{\lambda}^{\circ}$ be as before (on $D$ or on $X$ ). Then, if $\mathscr{L}$ is the nonlinear operator defined in (2.11),

TheOREM 7.1. Assume $\left\{\phi_{t}(\omega)\right\}$ satisfies $(2.1), E_{a}\left(\exp \left(-\phi_{t}\right)\right)$ is continuous in $t$ for all $a$, and that $S_{\lambda} 1(a)<\infty$ for all a. Then, $\hat{f}(x) \in \mathscr{D}(\bar{A})$ implies $f(a)-S_{\lambda}(\mathscr{L}-I) f(a)$ $\in \mathscr{D}(A)$ and

$$
\bar{A}_{\lambda} \hat{f}(a)=A_{\lambda}\left(I-S_{\lambda}(\mathscr{L}-I)\right) f(a) \text { in } D .
$$

Proof. If $\hat{f}(x) \in \mathscr{D}(\bar{A})$, then $\hat{f}(x)=\bar{R}_{\lambda} g(x)$ for some $g \in \mathscr{L}^{\infty}(X)$. Integrating (6.4) with this $g(x)$ and $a \in D$ yields

$$
\bar{T}_{t} g(a)=T_{t}^{\circ} g(a)+E_{a}\left[\chi_{[\tau \leqq t]} \int_{X} \bar{T}_{t-\tau} g(y) K\left(x_{\tau}, d y\right)\right]
$$

where $K(a, E)$ is the kernel defined by (6.2) if $n=1$. Arguing as in $\S 4$, with $K \bar{R}_{\lambda} g(a)$ considered as a function on $D$, we obtain

$R_{\lambda} g(a)=\left(I+S_{\lambda}\right)\left(I-S_{\lambda}^{\circ} K\right) \bar{R}_{\lambda} g(a), \quad \bar{A}_{\lambda} \hat{f}(a)=-g(a)=A_{\lambda}\left(I+S_{\lambda}\right)\left(I-S_{\lambda}^{\circ} K\right) \hat{f}(a)$.

Finally, $K \hat{f}(a)=\mathscr{L} f(a)$, and $S_{\lambda} \mathscr{L}|f|(a)<\infty$ by assumption. Equation (7.1) now follows as in (4.3).

CoRollary ([6]). If $\hat{f}(x) \in \mathscr{D}(\bar{A})$ and $x=\left(a_{1}, a_{2}, \ldots, a_{n}\right)$,

$$
\bar{A} \hat{f}(x)=\sum_{i=1}^{n} \bar{A} \hat{f}\left(a_{i}\right) \prod_{j \neq i} f\left(a_{j}\right), \quad \bar{A} \hat{f}(\partial)=0 .
$$

Proof. By (6.9) and the product rule for differentiation.

This proves the first part of Theorem 2.3. For the second part, assume $f(a)$ $-S_{\lambda}(\mathscr{L}-I) f(a) \in \mathscr{D}(A)$ for some $f(a) \in \mathscr{L}^{\infty}(D)$; we would like to show $\hat{f}(x) \in \mathscr{D}(\bar{A})$ on $X$. Our plan is first to show that a similar expression is in $\mathscr{D}(A)$ on $X$, and then 
apply arguments similar to those of Theorem 2.2 in $X$. First, however, we need objects analogous to $S_{\lambda} K g(x)$ and $S_{\lambda}^{\circ} K g(x)$, which cannot be the composition of two linear operators here. For simplicity, we assume that $\left\{\phi_{t}(\omega)\right\}$ is continuous in $t$ for $\omega$; this is not an essential restriction (see the remark after Lemma 7.1).

Lemma 7.1. Assume $\mu, K_{i}$ are as in (6.2), (6.3), $\left\{\phi_{t}\right\}$ as above, and $g \in \mathscr{L}^{\infty}(X)$, $g(x) \geqq 0$. Define

$$
\begin{aligned}
& S_{\lambda} \mu g(x)=E_{x}\left(\int_{0}^{\infty} e^{-\lambda s}\left(\sum_{i=1}^{n} K_{i} g\left(z_{s}\right) \frac{d \phi_{s}^{(i)}}{d \psi_{s}}\right) d \psi_{s}\right), \\
& S_{\lambda}^{\circ} \mu g(x)=E_{x}\left(e^{-\lambda \beta} \int_{X} g(y) \mu(w, d y)\right), \\
& S_{\lambda}^{\circ} \mu g(\partial)=S_{\lambda} \mu g(\partial)=0,
\end{aligned}
$$

where $\phi_{s}^{(i)}(w)=\phi_{s}\left(\omega_{i}\right)($ see $(6.1))$ and $d \phi_{s}^{(i)} / d \psi_{s}$ is the Radon-Nikodym derivative in $s$. Then, if $g(x)$ is as above and $f(a) \in \mathscr{L}^{\infty}(D), 0 \leqq f(a) \leqq 1, x=\left(a_{1}, a_{2}, \ldots, a_{n}\right)$,

$$
\begin{aligned}
S_{\lambda}(\mu-I) \hat{f}(x) & =\sum_{i=1}^{n} E_{a_{i}}\left(\int_{0}^{\infty} e^{-\lambda s}\left(\mathscr{L} f\left(x_{s}\right)-f\left(x_{s}\right)\right) \prod_{j \neq i}^{n} T_{s} f\left(a_{j}\right) d \phi_{s}\right), \\
S_{\lambda} \mu g(x) & =\sum_{n=1}^{\infty}\left(S_{\lambda}^{\circ}\right)^{n} \mu g(x) .
\end{aligned}
$$

Proof. First, by (6.2), (7.3), and the definition of $\mathscr{L}$,

$$
\begin{aligned}
S_{\lambda}(\mu-I) \hat{f}(x) & =\sum_{i=1}^{n} E_{x}\left(\int_{0}^{\infty} e^{-\lambda s}\left(K_{i} \hat{f}\left(z_{s}\right)-\hat{f}\left(z_{s}\right)\right) d \phi_{s}^{(i)}\right) \\
& =\sum_{i=1}^{n} E_{x}\left(\int_{0}^{\infty} e^{-\lambda s}\left(\mathscr{L} f\left(x_{s}^{(i)}\right)-f\left(x_{s}^{(i)}\right)\right) \prod_{j \neq i} f\left(x_{s}^{(j)}\right) d \phi_{s}^{(i)}\right) \\
& =\sum_{i=1}^{n} E_{a_{i}}\left(\int_{0}^{\infty} e^{-\lambda s}\left(\mathscr{L} f\left(x_{s}\right)-f\left(x_{s}\right)\right) \prod_{j \neq i} T_{s} f\left(a_{j}\right) d \phi_{s}\right)
\end{aligned}
$$

since the components $\left\{x_{s}^{(i)}\right\}$ of $z_{s}$ are independent. Second,

$$
\begin{aligned}
S_{\lambda}^{\circ} \mu g(x) & =\sum_{i=1}^{n} E_{x}\left(\exp \left(-\lambda \tau_{i}\right) \prod_{j \neq i} \chi_{\left[\tau_{j}>\tau_{i}\right]} K_{i} g\left(z_{\tau_{i}}\right)\right) \\
& =\sum_{i=1}^{n} E_{x}\left(\int_{0}^{\infty} e^{-\lambda s} K_{i} g\left(z_{s}\right)\left(\prod_{j \neq i} \exp \left(-\phi_{s}^{(j)}\right)\right) \exp \left(-\phi_{s}^{(i)}\right) d \phi_{s}^{(i)}\right) \\
& =E_{x}\left(\int_{0}^{\infty} e^{-\lambda s}\left(\sum_{i=1}^{n} K_{i} g\left(z_{s}\right) \frac{d \phi_{s}^{(i)}}{d \psi_{s}}\right) \exp \left(-\psi_{s}\right) d \psi_{s}\right) .
\end{aligned}
$$

By induction and $\S 3.2$ we deduce the same formula for $\left(S_{\lambda}^{\circ}\right)^{n+1} \mu g(x)$ except for a factor of $\psi_{s}^{n} / n$ ! in the integrand. Summation yields (7.5).

RemarK. Continuity of $\left\{\phi_{t}\right\}$ is not essential in Lemma 7.1; indeed if $\alpha_{t}^{(i)}=$ $\exp \left(-\phi_{t}^{(i)}\right)$ and $\gamma_{t}=\exp \left(-\psi_{t}\right)$ then Lemma 7.1 remains valid (by Lemma 3.3) 
provided $S_{\lambda} \mu g(x)$ is replaced by

$$
S_{\lambda} \mu g(x)=-E_{x}\left(\int_{0}^{\infty} e^{-\lambda s}\left(\sum_{i=1}^{n} K_{i} g\left(z_{s}\right) \frac{d \alpha_{s}^{(i)} / \alpha_{s}}{d \gamma_{s} / \gamma_{s}}\right) \frac{d \gamma_{s}}{\gamma_{s-}}\right)
$$

and similarly $d \phi_{s}$ is replaced by $d \alpha_{s} / \alpha_{s-}$ in (7.4).

TheOREM 7.2. Assume $\left\{\phi_{t}\right\}$ satisfies (2.1) and

$$
E_{a}\left(\phi_{t}\right) \leqq C_{t} \text { for all } a, \quad C_{t} \downarrow 0 .
$$

Assume also that $|f(a)| \leqq C<1, f(a)-S_{\lambda}(\mathscr{L}-I) f(a) \in \mathscr{D}(A)$ in $D$ for some $f(a)$ $\in \mathscr{L}^{\infty}(X)$. Then $\hat{f}(x)-S_{\lambda}(\mu-I) \hat{f}(x) \in \mathscr{D}(A)$ in $X$.

Proof. Note that (7.7) implies that $E_{a}\left(\exp \left(-\phi_{t}\right)\right)$ is continuous in $t$ uniformly in $a$. We assume for simplicity that $\left\{\phi_{t}\right\}$ is continuous, even though the proof goes through equally well in the discontinuous case. First, if $x=\left(a_{1}, a_{2}, \ldots, a_{n}\right), t>0$,

$$
\begin{aligned}
S_{\lambda}(\mu-I) \hat{f}(x) & =\sum_{i=1}^{n} E_{a_{i}}\left(\int_{0}^{\infty} e^{-\lambda s}\left(\mathscr{L} f\left(x_{s}\right)-f\left(x_{s}\right)\right) \prod_{j \neq i} T_{s} f\left(a_{j}\right) d \phi_{s}\right), \\
T_{t} S_{\lambda}(\mu-I) \hat{f}(x) & =\sum_{i=1}^{n} E_{a_{i}}\left(\theta_{t} \int_{0}^{\infty} e^{-\lambda s}\left(\mathscr{L} f\left(x_{s}\right)-f\left(x_{s}\right)\right) \prod_{j \neq i} T_{s+t} f\left(a_{j}\right) d \phi_{s}\right) \\
& =e^{\lambda t} \sum_{i=1}^{n} E_{a_{i}}\left(\int_{t}^{\infty} e^{-\lambda s}\left(\mathscr{L} f\left(x_{s}\right)-f\left(x_{s}\right)\right) \prod_{j \neq i} T_{s} f\left(a_{j}\right) d \phi_{s}\right) .
\end{aligned}
$$

Hence

$$
\begin{aligned}
&\left(T_{t}-I\right) S_{\lambda}(\mu-I) \hat{f}(x)=\left(e^{\lambda t}-1\right) S_{\lambda}(\mu-I) \hat{f}(x)-e^{\lambda t} \sum_{i=1}^{n} E_{a_{i}}\left(\int_{0}^{t} \cdots d \phi_{s}\right), \\
&\left(T_{t}-I\right)\left(I-S_{\lambda}(\mu-I)\right) \hat{f}(x)=-\left(e^{\lambda t}-1\right) S_{\lambda}(\mu-I) \hat{f}(x)+T_{t} \hat{f}(x)-\hat{f}(x) \\
&+e^{\lambda t} \sum_{i=1}^{n} E_{a_{i}}\left(\int_{0}^{t} e^{-\lambda s}\left(\mathscr{L} f\left(x_{s}\right)-f\left(x_{s}\right)\right) \prod_{j \neq i} T_{s} f\left(a_{j}\right) d \phi_{s}\right) .
\end{aligned}
$$

Fixing $n=1$ and using $f(a)-S_{\lambda}(\mathscr{L}-I) f(a) \in \mathscr{D}(A)$, we conclude

$$
\frac{1}{t}\left(T_{t} f(a)-f(a)+E_{a}\left(\int_{0}^{t}\left(\mathscr{L} f\left(x_{s}\right)-f\left(x_{s}\right)\right) d \phi_{s}\right)\right) \rightarrow C(a)
$$

where $C(a)=\lambda f(a)+A_{\lambda}\left(I-S_{\lambda}(\mathscr{L}-I)\right) f(a)$, and that the convergence is uniformly bounded for all $a \in D$ by some constant $M$. Now, if $|f(a)| \leqq C<1$, choose $C_{2}, t_{0}$ such that $C+2 E_{a}\left(\phi_{t_{0}}\right) \leqq C_{2}<1$. Then, if $0 \leqq t \leqq t_{0}$,

$$
\left|\prod_{1}^{n} T_{t} f\left(a_{i}\right)-\prod_{1}^{n}\left[f\left(a_{i}\right)-E_{a_{i}}\left(\int_{0}^{t}\left(\mathscr{L} f\left(x_{s}\right)-f\left(x_{s}\right)\right) d \phi_{s}\right)\right]\right| \leqq M \operatorname{tn} C_{2}^{n-1}
$$

Since we are only trying to show $\hat{f}-S_{\lambda}(\mu-I) \hat{f} \in \mathscr{D}(A)$, it is permissible to neglect terms on the right-hand side of (7.8) which (i) have a bound of the form $M t$ and (ii) are asymptotic (for all $x$ ) to an expression $C(x) t$, where $C(x) \in B_{0}$. However 
the expression in (7.9) is asymptotic to

$$
\left(\sum_{i=1}^{n} C\left(a_{i}\right) \prod_{j \neq i} f\left(a_{j}\right)\right) t
$$

by the product rule for differentiation. Hence the expression on the right-hand side of (7.8) is equivalent in the sense outlined above to

$$
\prod_{1}^{n}\left[f\left(a_{i}\right)-E_{a_{i}}\left(\int_{0}^{t}\left(\mathscr{L} f\left(x_{s}\right)-f\left(x_{s}\right)\right) d \phi_{s}\right)\right]-\prod_{1}^{n} f\left(a_{i}\right)
$$

$$
+\sum_{i=1}^{n} E_{a_{i}}\left[\int_{0}^{t}\left(\mathscr{L} f\left(x_{s}\right)-f\left(x_{s}\right)\right) \prod_{j \neq i}\left[f\left(a_{j}\right)-E_{a_{j}}\left(\int_{0}^{s}\left(\mathscr{L} f\left(x_{u}\right)-f\left(x_{u}\right)\right) d \phi_{u}\right)\right] d \phi_{s}\right] .
$$

The proof will be complete if we can show that this last expression is identically zero. First, expand (7.10) as a multinomial in the variables $\left\{f\left(a_{j}\right)\right\}$. If $S$ is a subset of the first $n$ integers of cardinality $n-l$, then the coefficient of $\prod_{s} f\left(a_{j}\right)$ in (7.10) will be $(-1)^{l}$ times

$$
\prod_{i=1}^{l} E_{b_{i}}\left(\int_{0}^{t} g\left(x_{s}\right) d \phi_{s}\right)-\sum_{i=1}^{l} E_{b_{i}}\left(\int_{0}^{t} g\left(x_{s}\right) \prod_{j \neq i} E_{b_{i}}\left(\int_{0}^{s} g\left(x_{u}\right) d \phi_{u}\right) d \phi_{s}\right)
$$

where $\left\{b_{1}, b_{2}, \ldots, b_{l}\right\}=\left\{a_{j}: j \notin S\right\}$ and $g(a)=(\mathscr{L}-I) f(a)$. We note that the integrand in the $i$ th term of the sum represents a random integral over the points $\left(s_{1}, s_{2}, \ldots, s_{l}\right) \in R^{l}$ with $0 \leqq s_{j} \leqq s_{i} \leqq t$ for $j \neq i$. Hence the expression is zero and (7.10) is identically zero. Q.E.D.

The proof of Theorem 2.3 is concluded by

THEOREM 7.3. Assume $\left\{\phi_{t}\right\}$ satisfies (2.1) and (7.7), and that $|f(a)| \leqq C<1$. Then, $f(a)-S_{\lambda}(\mathscr{L}-I) f(a) \in \mathscr{D}(A)$ in $D$ iff $\hat{f}(x) \in \mathscr{D}(\bar{A})$ on $X$.

Proof. First, note

$$
\begin{aligned}
S_{\lambda}^{\circ} 1(x) & =-E_{x}\left(\int_{0}^{\infty} e^{-\lambda s} d \exp \left(-\psi_{s}\right)\right)=\lambda \int_{0}^{\infty} e^{-\lambda s} E_{x}\left(1-\exp \left(-\psi_{s}\right)\right) d s \\
& \leqq \lambda \int_{0}^{\infty} e^{-\lambda s}\left(1-\exp \left(-n C_{s}\right)\right) d s=\beta_{\lambda, n}<1
\end{aligned}
$$

by (6.1), (7.7) and concavity of $\left(1-e^{-x}\right)$, and $S_{\lambda} 1(x) \leqq C_{\lambda, n}<\infty$ for all $x \in D^{n}$ by Theorem 3.1. Combining this and the identities (3.3), (7.5) we can now extend the proofs of Theorems $2.1,2.2$ in $\$ 4$ to fit our situation in $X$. In particular

$$
\bar{A}_{\lambda} \subseteq A_{\lambda}\left(I-S_{\lambda}(\mu-I)\right) \text { in } B
$$

where $B$ is any subspace of $B_{0}(X)$ which is preserved by the six operators $T_{t}, T_{t}^{\circ}, \bar{T}_{t}$, $R_{\lambda}, R_{\lambda}^{\circ}, \bar{R}_{\lambda}$. Moreover, we have equality in (7.12) iff there exist no nontrivial solutions of

$$
g(x)=E_{x}\left(e^{-\lambda \beta} \int_{X} g(y) \mu(w, d y)\right), \quad g(x) \in B
$$


Define

$$
B=\left\{g(x) \in B_{0}: \lim _{n \rightarrow \infty} \sup _{D^{n}}|g(y)|=0\right\} .
$$

Since $\sup _{D^{n}}\left|T_{t} g(x)\right| \leqq \sup _{D^{n}}|g(x)|$ etc., $T_{t}, R_{\lambda}, T_{t}^{\circ}, R_{\lambda}^{\circ}$ preserve $B$. I now claim that $\bar{T}_{t} g(x) \in B$ for any $g(x) \in B$ and $t>0$. By definition, if $g(x) \in B$, for any $\varepsilon>0$ there exists $M, n$ such that

and hence

$$
\begin{array}{ll}
|g(x)| \leqq M, & x \in \bigcup_{0}^{n-1} D^{l}, \\
|g(x)| \leqq \varepsilon, & x \in \bigcup_{n}^{\infty} D^{l},
\end{array}
$$

$$
\left|\bar{T}_{t} g(x)\right| \leqq \varepsilon+M \bar{P}_{x}\left(z_{t} \in \bigcup_{0}^{n-1} D^{l}\right) .
$$

If $x \in D^{m}, m>n>l$, then by Lemma 6.1

Now

$$
\bar{P}_{x}\left(z_{t} \in D^{l}\right)=\sum_{l_{1}+\cdots+l_{m}=l} \prod_{i=1}^{m} \bar{P}_{a_{i}}\left(z_{t} \in D^{l_{i}}\right) .
$$

$$
\bar{P}_{a}\left(z_{t}=\partial\right) \leqq \bar{P}_{a}(\beta \leqq t)=P_{a}(\tau \leqq t)=E_{a}\left(1-\exp \left(-\phi_{t}\right)\right)
$$

and $\bar{P}_{a}\left(z_{t}=\partial\right) \leqq\left(1-\exp \left(-C_{t}\right)\right)=d_{t}<1$ by concavity and (7.7). Hence, if $m \gg n>l$,

$$
\begin{aligned}
\bar{P}_{x}\left(z_{t} \in D^{l}\right) \leqq \sum_{0}^{l} j^{l-j}\left(\begin{array}{c}
m \\
j
\end{array}\right) d_{t}^{m-j}=\sum_{0}^{l} \frac{(m-j+1) \cdots(m-1) m}{j !} d_{t}^{m-j} j^{l-j}, \\
\bar{P}_{x}\left(z_{t} \in \bigcup_{0}^{n-1} D^{l}\right) \leqq m^{n+2} d_{t}^{m-n} n^{n} \leqq \varepsilon
\end{aligned}
$$

for sufficiently large $m$. Hence $\bar{T}_{t}$ preserves $B$ by (7.15), and since $\bar{R}_{\lambda}=\int_{0}^{\infty} e^{-\lambda t} \bar{T}_{t} f d t$, $\bar{R}_{\lambda}$ preserves $B$ also. Hence (7.12), (7.13) are applicable with $B$ given by (7.14). However, if (7.13) holds for $g(x) \in B$, and

$$
|g(x)| \leqq \frac{1}{2}\|g\|, \quad x \in \bigcup_{n}^{\infty} D^{l},
$$

where $\|g\|=\sup _{x}|g(x)|$, then by (7.11)

$$
\begin{aligned}
& |g(x)|=\left|S_{\lambda}^{\circ} \mu g(x)\right| \leqq S_{\lambda}^{\circ} 1(x)\|g\|, \\
& |g(x)| \leqq \beta_{\lambda, n}\|g\|, \quad x \in \bigcup_{0}^{n} D^{l} .
\end{aligned}
$$

Hence $\|g\|<\|g\|$ if $g \neq 0$, and (7.13) has no nontrivial solutions in $B$. Finally $\hat{f}(x) \in B$ if $|f(a)| \leqq C<1$, and by equality in (7.12), $\hat{f}(x)-S_{\lambda}(\mu-I) \hat{f}(x) \in \mathscr{D}(A)$ iff $\hat{f}(x) \in \mathscr{D}(\bar{A})$. Combining with Theorem 7.2, $\hat{f}(x) \in \mathscr{D}(\bar{A})$ iff $f(a)-S_{\lambda}(\mathscr{L}-I) f(a)$ $\in \mathscr{D}(A)$.

8. Branching diffusion processes. By basic results in PDE $[4$, p. 82], there exists a unique "Green's function" $p(t, x, y) \in C((0, \infty) \times \mathcal{O} \times \mathcal{O})$ satisfying the following 
two conditions. For fixed $t>0, y \in \mathcal{O}, p(t, x, y)$ has Hölder-continuous second partials in $x \in \overline{\mathcal{O}}$ and

$$
\begin{gathered}
\partial / \partial t p=A_{x} p ; \quad p\left(t, x_{0}, y\right)=0 \quad \text { for } x_{0} \in \partial \mathcal{O}, \\
p(t, x, y) \leqq\left(C / t^{n / 2}\right) \exp \left[-(\alpha / t)(x-y)^{2}\right], \quad \alpha>0 .
\end{gathered}
$$

Second, for $f(x) \in C_{0}(\mathcal{O})=\{f(x): f(x) \in C(\overline{\mathcal{O}}), f(x)=0$ on $\partial \mathcal{O}\}$,

$$
T_{t} f(x)=\int p(t, x, y) f(y) d y
$$

converges uniformly to $f(x)$ as $t \rightarrow 0$. By the interior Schauder estimates [4] we conclude

for all $y \in \mathcal{O}$.

$$
\begin{array}{rlrl}
\left|\partial / \partial x_{i} p(t, x, y)\right| & \leqq C_{t}(d), & & |x-\partial \mathcal{O}| \geqq d>0, \\
\left|\partial^{2} / \partial x_{i} \partial x_{j} p(t, x, y)\right| \leqq C_{t}(d), & & |x-\partial \mathcal{O}| \geqq d>0,
\end{array}
$$

Hence the operators $\left\{T_{t}\right\}$ map $\mathscr{L}^{\infty}(\mathcal{O})$ into $C^{2}(\mathcal{O}) \cap C_{0}(\mathcal{O})$, and $u(x, t)=T_{t} f(x)$ is the unique solution of $\partial / \partial t u=A_{x} u, u\left(x_{0}, t\right)=0$ on $\partial \mathcal{O}$ for $t>0$, and $u(x, 0)=f(x)$ for $f(x) \in C_{0}(\mathcal{O})$. In particular, by uniqueness, $\left\{T_{t}\right\}$ must be a semigroup, which implies in terms of $p(t, x, y)$ that

$$
p(t+s, x, y)=\int_{\mathcal{O}} p(s, x, z) p(t, z, y) d z .
$$

By (8.1) and the Hopf maximum principle, $\int p(1, x, z) d z \leqq \beta<1$; hence by (8.4) with $s=1$

$$
p(t, x, y) \leqq C^{\prime} \exp \left(-\alpha^{\prime} t\right), \quad t \geqq 1, \quad \alpha^{\prime}>0 .
$$

As a strongly continuous Feller semigroup on $C_{0}(\mathcal{O}),\left\{T_{t}\right\}$ is associated with a unique right-continuous strong Markov process [2, Chapter 3] $\left\{x_{t}\right\}$ on $\mathcal{O}$ such that $T_{t} f(x)=E_{x}\left[f\left(x_{t}\right)\right] ; P_{x}\left(x_{t} \in d y\right)=p(t, x, y) d y$. It can be shown that $\left\{x_{t}\right\}$ has continuous paths up to its termination time $\zeta(\omega)$, which here coincides with the first exit time from $\mathcal{O}$. The process $\left\{x_{t}\right\}$ does not remain in $\mathcal{O}$ indefinitely; indeed

by $(8.5)$.

$$
E_{x}(\zeta)=E_{x}\left(\int_{0}^{\infty} 1\left(x_{s}\right) d s\right)=\int_{0}^{\infty} \int_{\mathcal{O}} p(t, x, y) d y d t \leqq C^{\prime \prime}
$$

Define

$$
f(t, x)=\int_{0}^{t} \int_{\Gamma} p(s, x, y) \beta(y) \Sigma(d y) d s,
$$

where $\Gamma, \beta(y)$ are as in Theorem 2.5 and $\Sigma(d y)$ is surface measure on $\Gamma$. Breaking $\Gamma$ up into coordinate patches and applying (8.2), (8.4), we obtain $0 \leqq f(t, x) \leqq C^{m} \sqrt{ } t$, $f(t+s, x)=f(t, x)+T_{t} f(s, \cdot)(x)$. Hence there exists [2, Chapter 6] a unique continuous additive functional $\left\{\phi_{t}\right\}$ satisfying (2.1), (2.2), and

$$
E_{x}\left(\phi_{t}\right)=\int_{0}^{t} \int_{\Gamma} p(s, x, y) \beta(y) \Sigma(d y) d s \leqq C^{m} \sqrt{ } t .
$$


This is "local time on $\Gamma$ with weight $\beta(x)$." In particular

$$
S f(x)=E_{x}\left(\int_{0}^{\infty} f\left(x_{s}\right) d \phi_{s}\right)=\int_{0}^{\infty} \int_{\Gamma} p(s, x, y) f(y) \beta(y) \Sigma(d y) d s
$$

converges uniformly for any $f(x) \in \mathscr{L}^{\infty}(\Gamma)$. If $y \in \Gamma,|x-\partial \mathcal{O} \cup \Gamma| \geqq d>0$, then by the Schauder estimates again and (8.2), (8.5), we can take $C_{t}(d)=C \exp (-\alpha t-\gamma / t)$ in (8.3). Hence by (8.1) and an integration by parts

$$
S f(x) \in C^{2}(\mathcal{O}-\Gamma), \quad A_{x} S f(x)=0 \quad \text { in } \mathcal{O}-\Gamma .
$$

We now investigate the behavior of $S f(x)$ near $\Gamma$. Note

$$
S f(x)=\int_{0}^{1} \int_{\Gamma} p(s, x, y) f(y) \beta(y) \Sigma(d y) d s+\int_{\mathcal{O}} S f(y) p(1, x, y) d y
$$

and let $q(t, x, y)$ be the fundamental solution of $\partial / \partial t q=A_{x} q$ in $R^{n}$ [4, Chapter 1]. Thus $0 \leqq p(t, x, y) \leqq q(t, x, y)$, and by the construction of $p(t, x, y)$

$$
\begin{aligned}
|p(t, x, y)-q(t, x, y)| & \leqq C e^{-\gamma / t}, \\
\left|\partial / \partial x_{i} p(t, x, y)-\partial / \partial x_{i} q(t, x, y)\right| & \leqq C e^{-\gamma / t}, \\
\left|\partial^{2} / \partial x_{i} \partial x_{j} p(t, x, y)-\partial^{2} / \partial x_{i} \partial x_{j} q(t, x, y)\right| & \leqq C e^{-\gamma / t},
\end{aligned}
$$

for $|x-\partial \mathcal{O}| \geqq d>0,|y-\partial \mathcal{O}| \geqq d>0, \gamma=\gamma(d)>0$. Hence

$$
S f(x)-\int_{0}^{1} \int_{\Gamma} q(s, x, y) f(y) \beta(y) \Sigma(d y) d s \in C^{2}(\mathcal{O}) .
$$

Now the integral above is a single-layer potential of $q(t, x, y)$ on $\Gamma$, and as such is known to satisfy a certain jump condition across $\Gamma$. More precisely, let

$$
D_{v}^{ \pm}=\sum_{1}^{n} \sum_{1}^{n} a_{i j}(x) n_{i}^{ \pm}(x) \partial / \partial x_{j}
$$

by the two outwards-pointing conormal derivatives for $x \in \Gamma$, where $\left\{n_{i}^{ \pm}(x)\right\}$ are orthonormal vectors to $\Gamma$ at $x$. Then [4, Chapter 5]

$$
\frac{1}{2}\left(D_{v}^{+}+D_{v}^{-}\right) S f(x)=-\beta(x) f(x)
$$

for all $x \in \Gamma$ and $f(x) \in C(\Gamma)$.

Finally, let $K(x, A)$ be a Feller substochastic kernel on $\mathcal{O}$. By Theorem 2.4 and (8.6), there exists a unique semigroup $\left\{\bar{T}_{t}\right\}$ on $C_{0}(\mathcal{O})$ satisfying (2.5) and (see also Theorem 5.3)

$$
\bar{A}=A(I-S(K-I)) .
$$

THEOREM 8.1. $f(x) \in \mathscr{D}(\bar{A})$ iff $f(x)=g(x)+k(x)$, where $g(x) \in \mathscr{D}(A), k(x) \in C_{0}(\mathcal{O})$ $\cap C^{2}(\mathcal{O}-\Gamma), A_{x} k(x)=0$ in $\mathcal{O}-\Gamma$, and $f(x)=g(x)+k(x)$ satisfies for $x \in \Gamma$

$$
\frac{1}{2}\left(D_{v}^{+}+D_{v}^{-}\right) f(x)=-\beta(x)\left(\int_{\mathcal{O}} f(y) K(x, d y)-f(x)\right) .
$$


Proof. First, by (8.11), $f(x) \in \mathscr{D}(\bar{A})$ iff $f(x)-S(K-I) f(x) \in \mathscr{D}(A)$, or iff $f(x)$ $=g(x)+S(K-I) f(x), g(x) \in \mathscr{D}(A)$. If $f(x) \in \mathscr{D}(\bar{A})$, then, by (8.7), (8.10), $k(x)$ $=S(K-I) f(x)$ satisfies the conditions stated. Conversely, if $f(x)=g(x)+k(x)$ as above, let $l(x)=k(x)-S(K-I) f(x)$. Then $l(x) \in C_{0}(\mathcal{O}) \cap C^{2}(\mathcal{O}-\Gamma), A_{x} l(x)=0$ in $\mathcal{O}-\Gamma$, and $\frac{1}{2}\left(D_{v}^{+}+D_{v}^{-}\right) l(x)=0$ on $\Gamma$. Hence if $l\left(x_{0}\right)=\sup _{\mathcal{O}} l(y)>0$, we must have $x_{0} \in \Gamma$; but if $l(y)<l\left(x_{0}\right)$ for all $y \in \mathcal{O}-\Gamma$ and $x_{0} \in \Gamma$, then $D_{v}^{ \pm} l\left(x_{0}\right)<0[4$, Theorems 2.1, 2.14]. Hence $l(x) \equiv 0, k(x)=S(K-I) f(x)$, and $f(x) \in \mathscr{D}(\bar{A})$ by (8.11).

A useful corollary of Theorem 8.1 is

COROLlaRy 8.1. $f(x) \in \mathscr{D}(\bar{A})$ iff $f(x)=g(x)+k(x)$, where $g(x) \in \mathscr{D}(A), k(x) \in C_{0}(\mathcal{O})$ $\cap C^{2}(\mathcal{O}-\Gamma), f(x)$ satisfies (8.12), and $A_{x} k(x)$, defined on $\mathcal{O}-\Gamma$, extends to some function in $C_{0}(\mathcal{O})$.

THEOREM 8.2. If $f(x) \in C(\overline{\mathcal{C}})$, then $u(x, t)=\bar{T}_{t} f(x)$ is the unique solution of the system (2.20), (2.21).

Proof. Uniqueness follows from maximum principle arguments as in the proof of Theorem 8.1. For existence, note that by (4.1), (4.3),

$$
\bar{R}_{\lambda} f(x)=R_{\lambda} f(x)+S_{\lambda}(K-I) \bar{R}_{\lambda} f(x) .
$$

Inverting Laplace transforms

$$
\bar{T}_{t} f(x)=T_{t} f(x)+\int_{0}^{t} \int_{\Gamma} p(s, x, y)(K-I) \bar{T}_{t-s} f(y) \beta(y) \Sigma(d y) d s .
$$

In particular $\bar{T}_{t} f(x) \in C_{0}(\mathcal{O}) \cap C^{2}(\mathcal{O}-\Gamma)$, and since $\left\{\bar{T}_{t}\right\}$ is strongly continuous, $\frac{1}{2}\left(D_{v}^{+}+D_{v}^{-}\right) \bar{T}_{t} f(x)=-\beta(x)(K-I) \bar{T}_{t} f(x)$ by (8.8), (8.13). Also, assume $|x-\partial \mathcal{O}|$ $\geqq d>0,|x-\Gamma| \geqq d>0$. Then by (8.2), (8.8), (8.13)

$$
\left|\bar{T}_{s} h(x)-\int q(s, x, y) h(y) d y\right| \leqq C \exp (-\gamma / s)
$$

for any $h(x) \in \mathscr{L}^{\infty}\left(R^{n}\right)$. However, if $h(x)$ is twice continuously differentiable in a neighborhood of a point $x_{0} \in R^{n}$, then

$$
\frac{1}{s} \int q\left(s, x_{0}, y\right)\left(h(y)-h\left(x_{0}\right)\right) d y \rightarrow A_{x} h\left(x_{0}\right)
$$

by Taylor's formula (see [4, Chapter 1]). If $h(x)=\bar{T}_{t} f(x)$ for some $t>0$, we conclude $\partial / \partial t \bar{T}_{t} f(x)=A_{x} \bar{T}_{t} f(x)$ in $\mathcal{O}-\Gamma$. Q.E.D.

REMARK. According to Corollary 8.1, the relations

$$
\begin{aligned}
& T_{t}: \mathscr{L}^{\infty}(\mathcal{O}) \rightarrow \mathscr{D}(A), \quad \text { all } t>0, \\
& T_{t}^{\circ}: \mathscr{L}^{\infty}(\mathcal{O}) \rightarrow \mathscr{D}\left(A^{\circ}\right), \quad \text { all } t>0 \text {, } \\
& \bar{T}_{t}: \mathscr{L}^{\infty}(\mathcal{O}) \rightarrow \mathscr{D}(\bar{A}), \quad \text { all } t>0 \text {, }
\end{aligned}
$$

follow as soon as we can show that e.g., $A_{x} \bar{T}_{t} f(x)$ is well behaved near $\partial \mathcal{O}$ and $\Gamma$ for any $f(x) \in C_{0}(\mathcal{O})$. This can be done by iteration in (8.13) and standard potential arguments if e.g., (i) $|\partial / \partial t p(t, x, y)| \leqq C(t), x, y \in \mathcal{O}$, and (ii) $K(x, N)=0$ for all 
$x \in \Gamma$ and some neighborhood $N$ of $\partial \mathcal{O}$. Condition (i) seems reasonable, but we have not been able to remove (ii).

8.1. We assume the hypotheses of Theorem 2.6. According to S. Itô [8], as formulated by Sato and Ueno [17] in the time-homogeneous case, there exists a unique function $p(t, x, y) \in C((0, \infty) \times \overline{\mathcal{O}} \times \overline{\mathcal{O}})$ satisfying the following two conditions. First, $p(t, x, y)$ is twice continuously differentiable for $x, y \in \overline{\mathcal{O}}$ and any $t>0$, and

$$
\partial / \partial t p=A_{x} p \quad \text { in } \mathcal{O}, \quad D_{v(x)} p=0 \quad \text { on } \partial \mathcal{O}
$$

where $D_{v}$ is the inwards-pointing conormal derivative at $x \in \partial \mathcal{O}$ defined by (8.9). Second, if $f(x) \in C(\overline{\mathcal{O}})$,

$$
u(x, t)=T_{t} f(x)=\int_{\mathcal{O}} f(y) p(t, x, y) d y
$$

converges uniformly to $f(x)$ as $t \rightarrow 0$. Thus $u(x, t)$ is the unique solution of $\partial / \partial t u=A_{x} u$ in $\mathcal{O}, D_{v} u(x, t)=0$ on $\partial \mathcal{O}(t>0)$ and $u(x, 0)=f(x)$. By uniqueness, $\left\{T_{t}\right\}$ is a strongly continuous Feller semigroup on $C(\overline{\mathcal{O}})$, and, as before, there exists a unique strong Markov process $\left\{x_{t}\right\}$ on $\overline{\mathcal{O}}$ such that $T_{t} f(x)=E_{x}\left[f\left(x_{t}\right)\right]$.

It follows from the construction of $p(t, x, y)$ and standard arguments (see e.g. $[8$, p. 308], [23], (8.2), (8.8)) that

$$
\int_{\partial \mathcal{O}} p(t, x, y) \Sigma(d y) \leqq C / \sqrt{ } t, \quad \int_{\partial \mathcal{O}}\left|\partial / \partial x_{i} p(t, x, y)\right| \Sigma(d y) \leqq C / t
$$

for all $x \in \overline{\mathcal{O}}$ and $t \leqq 1$. Hence by the inequality $\min \{b, a b\} \leqq b a^{\alpha}, 0<\alpha<1$, and the Mean Value Theorem

$$
\int_{\partial \mathcal{O}}\left|p\left(t, x_{1}, y\right)-p\left(t, x_{2}, y\right)\right| \Sigma(d y) \leqq \frac{C}{\sqrt{t^{1+\alpha}}}\left|x_{1}-x_{2}\right|^{\alpha}
$$

for all $\alpha, 0<\alpha<1$. In particular, by (8.16) and [2, Chapter 6], there exists a unique additive functional $\left\{\phi_{t}\right\}$ satisfying (2.1), (2.2), and

$$
E_{x}\left(\phi_{t}\right)=\int_{0}^{t} \int_{\partial \mathcal{O}} p(s, x, y) \beta(y) \Sigma(d y) d s \leqq C \sqrt{ } t .
$$

Moreover, given any $g(s, x) \in C([0, t] \times \overline{\mathcal{O}}) \cap C^{\alpha}([\varepsilon, t] \times \overline{\mathcal{O}})$ for all $\varepsilon>0$, some $\alpha>0$, then [17, Theorem 2.2]

$$
v(x, t)=E_{x}\left(\int_{0}^{t} g\left(t-s, x_{s}(\omega)\right) d \phi_{s}\right)=\int_{0}^{t} \int_{\partial \mathcal{O}} p(s, x, y) g(t-s, y) \beta(y) \Sigma(d y) d s
$$

is of class $C^{2}(\mathcal{O}) \cap C^{1}(\overline{\mathcal{O}})$ for fixed $t>0$ and satisfies

$$
\left(A_{x}-\partial / \partial t\right) v=0 \quad \text { in } \mathcal{O}, \quad D_{v(x)} v(x, t)=-\beta(x) g(t, x) \quad \text { on } \partial \mathcal{O} .
$$

In particular, if $f(x) \in C^{\alpha} \partial(\mathcal{O})$,

$$
\begin{aligned}
S_{\lambda} f(x) & =E_{x}\left(\int_{0}^{\infty} e^{-\lambda s} f\left[x_{s}(\omega)\right] d \phi_{s}\right) \\
& =\int_{0}^{1} \int_{\partial \odot} e^{-\lambda s} p(s, x, y) f(y) \beta(y) \Sigma(d y) d s+e^{-\lambda} T_{1} S_{\lambda} f(x)
\end{aligned}
$$


satisfies

$$
\begin{gathered}
S_{\lambda} f(x) \in C^{2}(\mathcal{O}) \cap C^{1}(\overline{\mathcal{O}}), \quad\left(A_{x}-\lambda\right) S_{\lambda} f(x)=0, \\
D_{v(x)} S_{\lambda} f(x)=-\beta(x) f(x) \quad \text { on } \partial \mathcal{O} .
\end{gathered}
$$

Finally, we remark [17, Lemma 2.2 , p. 600] that $\mathscr{D}(A) \subseteq C^{1}(\overline{\mathcal{O}})$; indeed, if $g(x)$ $\in \mathscr{D}(A)$, then $g(x) \in C^{1}(\overline{\mathcal{O}})$ and $D_{v(x)} g \equiv 0$ on $\partial \mathcal{O}$.

Now, if $\left\{p_{n}(a)\right\}$ satisfy $(2.22)$, let $\pi_{n}(a, E)=p_{n}(a) \chi_{E}((a, a, \ldots, a))$ and construct the branching process (see $\S 6)$ corresponding to $\left\{x_{t}\right\}$ on $\overline{\mathcal{O}},\left\{\phi_{t}\right\}$, and $\left\{\pi_{n}\right\}$. By construction of $\left\{\phi_{t}\right\}$, all branching must take place on $\partial \mathcal{O}$, and $\mathscr{L} f(a)=\sum_{0}^{\infty} p_{n}(a) f(a)^{n}$.

THEOREM 8.3. If $f(a) \in \mathscr{L}^{\infty}(\overline{\mathcal{O}})$ and $|f(a)| \leqq c<1$, then $\hat{f}(x) \in \mathscr{D}(\bar{A})$ iff $f(a)=g(a)$ $+k(a)$, where $g(a) \in \mathscr{D}(A), k(a) \in C^{2}(\mathcal{O}) \cap C^{1}(\overline{\mathcal{O}}),\left(A_{a}-\lambda\right) k(a)=0$ for some $\lambda>0$, and, for $a \in \partial \mathcal{O}, f(a)=g(a)+k(a)$ satisfies

$$
D_{v} f(a)=-\beta(a)\left(\sum_{0}^{\infty} p_{n}(a) f(a)^{n}-f(a)\right) .
$$

Proof. By Theorem 2.3, $\hat{f}(x) \in \mathscr{D}(\bar{A})$ iff $f(a)-S_{\lambda}(\mathscr{L}-I) f(a) \in \mathscr{D}(A)$, or iff $f(a)=g(a)+S_{\lambda}(\mathscr{L}-I) f(a), g(a) \in \mathscr{D}(A)$. I claim $k(a)=S_{\lambda}(\mathscr{L}-I) f(a)$ satisfies the conditions above. First, since evidently $(\mathscr{L}-I) f(a) \in \mathscr{L}^{\infty}(\partial \mathcal{O}), k(a) \in C^{\alpha}(\overline{\mathcal{O}})$ by (8.17). Since $f(a)=g(a)+k(a), f(a) \in C^{\alpha}(\overline{\mathcal{O}})$. Thus $(\mathscr{L}-I) f(a) \in C^{\alpha}(\partial \mathcal{O})$ by $(2.22$ (ii)), and by (8.19), $S_{\lambda}(\mathscr{L}-I) f(a) \in C^{2}(\mathcal{O}) \cap C^{1}(\overline{\mathcal{O}}),\left(A_{a}-\lambda\right) S_{\lambda}(\mathscr{L}-I) f(a)=0$, and $D_{v} k(a)=-\beta(a)(\mathscr{L}-I) f(a)$. Conversely, if $f(a)=g(a)+k(a)$ as above, let $l(a)$ $=k(a)-S_{\lambda}(\mathscr{L}-I) f(a)$. Then $\left(A_{a}-\lambda\right) l(a)=0$ in $\mathcal{O}, D_{v} l(a)=0$ on $\partial \mathcal{O}$, and $l(a) \equiv 0$ as before. Hence $g(a)=f(a)-S_{\lambda}(\mathscr{L}-I) f(a) \in \mathscr{D}(A)$ and $\hat{f}(x) \in \mathscr{D}(\bar{A})$ by Theorem 2.3.

THEOREM 8.4. Assume $f(a) \in C(\overline{\mathcal{O}})$ and $|f(a)| \leqq c<1$. Then, $u(a, t)=\bar{E}_{a}\left(\hat{f}\left(x_{t}\right)\right)$ is the unique solution of (2.23), (2.24).

Proof. By arguing as in $\S 4, R_{\lambda} f(a)=\left(I-S_{\lambda}(K-I)\right) \bar{R}_{\lambda} \hat{f}(a)$ where $K(a, A)$ is defined in $\S 6$. Thus by the branching property (so that $K \bar{T}_{t} \hat{f}(a)=\mathscr{L} \bar{T}_{t} \hat{f}(a)$ ) and uniqueness of the Laplace transform

$$
\bar{T}_{t} \hat{f}(a)=T_{t} f(a)+\int_{0}^{t} \int_{\partial \mathcal{O}} p(s, a, b)(\mathscr{L}-I) \bar{T}_{t-s} \hat{f}(b) \beta(b) \Sigma(d b) d s .
$$

Thus $\bar{T}_{t} \hat{f}(a) \in C^{\alpha}(\overline{\mathcal{O}})$ and $\bar{T}_{t} \hat{f}(a) \in C^{2}(\mathcal{O}) \cap C^{1}(\overline{\mathcal{O}})$ by (8.17), (8.18), and (8.20) (i.e., (2.24)) follows for $\bar{T}_{t} \hat{f}(a)$. The proof that $\left(A_{a}-\partial / \partial t\right) \bar{T}_{t} \hat{f}(a)=0$ for $t>0, a \in \mathcal{O}$, proceeds as in Theorem 8.2. To prove uniqueness, let $u(a, t)$ be a solution of (2.23), (2.24) for $0 \leqq t \leqq T$ with $u(a, t) \in C^{2}(\mathcal{O}) \cap C^{1}(\overline{\mathcal{O}}), 0<t \leqq T$. First, I claim $|u(a, t)|$ $\leqq C^{\prime}<1, a \in \overline{\mathcal{O}}, 0 \leqq t \leqq T$. If not, there exist $a_{0} \in \partial \mathcal{O}, t_{0}>0$, such that $|u(a, t)|<1$ for $t<t_{0}, a \in \overline{\mathcal{O}}$, and $\left|u\left(a_{0}, t_{0}\right)\right|=1$. If $u\left(a_{0}, t_{0}\right)=+1$, then $D_{v} u\left(a_{0}, t_{0}\right)<0$ (see [4, Theorem 2.14]) and $(\mathscr{L}-I) u\left(a_{0}, t_{0}\right)=0$, which contradicts (2.24). If $u\left(a_{0}, t_{0}\right)=-1$, then $D_{v} u\left(a_{0}, t_{0}\right)>0$ while $(\mathscr{L}-I) u\left(a_{0}, t_{0}\right)=\Sigma p_{n}\left(a_{0}\right)(-1)^{n}+1 \geqq 0$. Hence $|u(a, t)|$ $\leqq C^{\prime}<1,0 \leqq t \leqq T$. Finally, set $v(a, t)=u(a, t)-S_{\lambda}(\mathscr{L}-I) u(a, t)$. Then $v(a, t)$ 
$\in C^{2}(\mathcal{O}) \cap C^{1}(\overline{\mathcal{O}})$ and $D_{v(a)} v(a, t)=0$ on $\partial \mathcal{O}$ for $0<t \leqq T$; hence $v(a, t) \in \mathscr{D}(A)$ for these $t$ [23, Theorem 2]. Thus $\hat{u}(x, t) \in \mathscr{D}(\bar{A})$ and $(\bar{A}-\partial / \partial t) \hat{u}(x, t)=0$ by Theorem 2.3. Since $\hat{u}(x, 0)=\hat{f}(x), \hat{u}(x, t)=\bar{T}_{t} \hat{f}(x)$ for $0 \leqq t \leqq T$ by standard arguments.

9. A converse of Theorem 2.1. A process $\left\{x_{t}, B_{t}\right\}$ is said to be a Hunt process if (i) it is a strong Markov process with right-continuous paths, (ii) each sample path $\left\{x_{t}(\omega)\right\}$ has a left-limit $\left\{x_{t-}(\omega)\right\}$ at all $t$, and (iii) $\left\{x_{t}, B_{t}\right\}$ is quasi-left continuous, i.e., if $\left\{\beta_{n}>t\right\} \in B_{t}$ for all $t$ and $\beta_{n} \uparrow \beta$, then $P_{x}\left(x_{\beta_{n}} \rightarrow x_{\beta}, \beta<\infty\right)=P_{x}(\beta<\infty)$. If $X$ is a locally compact metric space, such processes can be constructed corresponding to any strongly-continuous Markovian semigroup $\left\{T_{t}\right\}$ on

$$
C_{0}(X)=\left\{f(x) \in B C(X): \lim _{x \rightarrow \infty} f(x)=0\right\}
$$

(see [2, Chapter 3]). We will also make use of the condition $L$ of Meyer [10, p. 160], which can perhaps be most perspicuously stated as [10], [12]

(L) There exists a nonnegative finite measure $\mu$ on $X$ such that if $\tau(A)=\sup \left\{t: x_{t}(\omega) \notin A\right\}$ for some " nearly Borel" set $A$, then $P_{\mu}(\tau(A)<\infty)$ $=\int_{X} P_{y}(\tau(A)<\infty) \mu(d y)=0$ implies $P_{x}(\tau(A)<\infty) \equiv 0$.

By a generalized continuous additive functional (gcaf) we will mean a continuous additive functional of the type considered in $\$ 3.1$, i.e., satisfying (2.1), (2.2) with (2.1 (ii)) replaced by (a) $\phi_{0}(\omega)=0,0 \leqq \phi_{t}(\omega) \leqq \infty$ and (b) $\phi_{t}(\omega)=\infty$ implies $\phi_{t-}(\omega)=\infty$, and (2.2) interpreted in the sense of continuity in the extended real numbers. By Theorem 3.2 and $\S 4$, such $\left\{\phi_{t}\right\}$ can be used in Theorems 2.1, 2.2, with (2.8) replaced by (4.2).

Our result is

THEOREM 9.1. Let $\left\{x_{t}, B_{t}\right\}$ be a Hunt process satisfying condition (L) on a locally compact metric space $X$, and assume that almost every sample path of $x_{t}(\omega)$ is continuous except for a discrete set of jumps. Then, there exists another strong Markov process $\left\{y_{t}, N_{t}\right\}$ on $X$, which has continuous paths up to its termination time, and a gcaf $\left\{\phi_{t}\right\}$ of $\left\{y_{t}\right\}$ and substochastic kernel $K(x, A)$ such that

$$
T_{t} f(x)=T_{t}^{\circ} f(x)+E_{x}^{\prime \prime}\left[\chi_{[\tau \leqq t]} \int_{x} T_{t-\tau} f(z) K\left(y_{\tau}, d z\right)\right]
$$

for $f(x) \in \mathscr{L}^{\infty}(X)$, where $P_{x}^{\prime \prime}\left(\tau>t \mid N_{\infty}\right)=\exp \left(-\phi_{t}\right)$ and $T_{t}^{\prime \prime} f(x)=E_{x}^{\prime \prime}\left[f\left(y_{t}\right)\right], T_{t}^{\circ} f(x)$ $=E_{x}^{\prime \prime}\left[f\left(y_{t}\right) \chi_{[\tau>t]}\right]$. Moreover, for fixed $\left\{y_{t}\right\}$ and $K,\left\{T_{t}\right\}$ is the unique Markovian solution of (9.1), and hence we have equality in (4.2) or (9.7).

REMARK. That $\left\{x_{t}(\omega)\right\}$ have discrete jumps a.s. is not essential; all that is required is that there exists a Markov time $\tau_{1}(\omega)$ such that if $\tau_{n+1} \equiv \tau_{n}+\theta_{\tau_{n}} \tau_{1}$ then $P_{x}\left[\tau_{n} \rightarrow \infty\right] \equiv 1$. We would then conclude that the process $\left\{y_{t}\right\}$ was continuous at the analogue of the $\left\{\tau_{n}\right\}$. Also, one can construct simple examples where the gcaf $\left\{\phi_{t}\right\}$ obtained from Theorem 9.1 is not of the form (2.1), (2.2). 
Proof. Set $\tau_{1}(\omega)=\inf \left\{t: x_{t}(\omega) \neq x_{t-}(\omega)\right\}, \quad \tau_{2}=\tau_{1}+\theta_{\tau_{1}} \tau_{1}$ and for all $n \geqq 2$, $\tau_{n+1}=\tau_{n}+\theta_{\tau_{n}} \tau_{1}$. Thus $\tau_{n}(\omega)$ is the $n$th jumping time of $x_{t}(\omega)$; as the remark indicates, we could have made it the $n$th jump of amplitude $\geqq \varepsilon$ (note $\tau_{n+1}=\infty$ by definition if $\left.\tau_{n} \geqq \zeta\right)$. Define $T_{t}^{\circ} f(x)=E_{x}\left[f\left(x_{t}\right) \chi_{\left[\tau_{1}>t\right]}\right]$ and, as in Moyal [13], let $\left\{T_{t}^{\prime \prime}\right\}$ be the minimal solution of

$$
T_{t}^{\prime \prime} f(x)=T_{t}^{\circ} f(x)+E_{x}\left[\chi_{\left[\tau_{1} \leqq t\right]} T_{t-\tau_{1}}^{\prime \prime} f\left(x_{\tau_{1}-}\right)\right] .
$$

As the construction of Ikeda-Nagasawa-Watanabe [6, II, Theorem 2.2] indicates, there exists a model $\left\{y_{t}, N_{t}\right\}$ of $\left\{T_{t}^{\prime \prime}\right\}$ which has continuous paths strictly before its termination time and is a strong Markov process. Since $0 \leqq T_{t}^{\circ} f(x) \leqq T_{t}^{\prime \prime} f(x)$ if $f(x) \geqq 0$ by (9.2), there also exists a contractive multiplicative functional $\left\{\alpha_{t}\right\}$ of $\left\{y_{t}\right\}$ such that

$$
T_{t}^{\circ} f(x)=E_{x}^{\prime \prime}\left[f\left(y_{t}\right) \alpha_{t}\right]=E_{x}^{\prime \prime}\left[f\left(y_{t}\right) \chi_{[\tau>t]}\right]
$$

where $P_{x}^{\prime \prime}\left[\tau>t \mid N_{\infty}\right]=\alpha_{t}$ [2, Theorem 9.3]. By (9.3) we may as well assume $\alpha_{t}(\omega) \equiv \alpha_{t+}(\omega)$ and $\alpha_{t}(\omega) \equiv \alpha_{\zeta(\omega)-}(\omega)$ for $t \geqq \zeta(\omega)$. I now claim $\alpha_{t}(\omega)=\exp \left(-\phi_{t}(\omega)\right)$ for some gcaf $\left\{\phi_{t}\right\}$; all that is necessary is to prove $\alpha_{t}(\omega) \in C([0, \infty))$ a.s. $P_{x}^{\prime \prime}$. However, if $\beta(\omega)=\inf \left\{t: \alpha_{t-}(\omega)-\alpha_{t}(\omega) \geqq \varepsilon\right\}$ for some $\varepsilon>0$, then either $\beta(\omega)=\infty$ or $\beta(\omega)<\zeta(\omega)$, in which case $y_{t}(\omega)$ is continuous at $\beta(\omega)$. Hence $[11$, p. 118] $\beta(\omega)$ is accessible, and there exist stopping times $\beta_{n} \uparrow \beta, \beta_{n}<\beta$, such that

$$
\begin{aligned}
E_{x}^{\prime \prime}\left[\chi_{[\beta<\infty]}\left(\alpha_{\beta-}-\alpha_{\beta}\right)\right] & =P_{x}^{\prime \prime}[\tau=\beta<\infty] \\
& =P_{x}^{\prime \prime}\left[\beta_{n}<\tau, \beta_{n} \uparrow \tau<\infty\right]=P_{x}^{\circ}\left[\beta_{n}<\zeta^{\circ}, \beta_{n} \uparrow \zeta^{\circ}<\infty\right] \\
& =P_{x}\left[\beta_{n}<\tau_{1}, \beta_{n} \uparrow \tau_{1}<\infty\right]=0
\end{aligned}
$$

since $\left\{x_{t}\right\}$ is quasi-left continuous and $x_{\tau_{1}} \neq x_{\tau_{1}-}$ a.s. Hence $\beta(\omega)=\infty$ a.s. Q.E.D.

By a theorem of Watanabe [22], there exists a nonnegative continuous additive functional $\left\{\sigma_{t}(\omega)\right\}$ of $\left\{x_{i}\right\}$ and a kernel $n(x, A)(0 \leqq n(x, A) \leqq \infty)$ such that for all Borel functions $f(x, y) \geqq 0$

$$
E_{x}\left(\sum_{1}^{\infty} e^{-\lambda \tau_{n}} f\left(x_{\tau_{n}-}, x_{\tau_{n}}\right)\right)=E_{x}\left(\int_{0}^{\infty} e^{-\lambda s} \int_{X} f\left(x_{s}, y\right) n\left(x_{s}, d y\right) d \sigma_{s}\right) .
$$

It follows from the construction in [22] that we also have

(i) For any stopping time $\beta(\omega)$ of $\left\{x_{t}\right\}$

$$
E_{x}\left(\sum_{\tau_{n} \leqq \beta} \exp \left(-\lambda \tau_{n}\right) f\left(x_{\tau_{n^{-}}}, x_{\tau_{n}}\right)\right)=E_{x}\left(\int_{0}^{\beta} e^{-\lambda s} \int_{X} f\left(x_{s}, y\right) n\left(x_{s}, d y\right) d \sigma_{s}\right) \text {. }
$$

(ii) There exists sets $\Gamma_{m} \uparrow X$ for which $n\left(x, \Gamma_{m}\right) \leqq m$.

In particular, if $\beta=\tau_{1}$ and $A \subseteq \Gamma_{m}$,

$$
\begin{aligned}
E_{x}\left(\exp \left(-\lambda \tau_{1}\right) \chi_{A}\left(x_{\tau_{1}}\right)\right) & =E_{x}\left(\int_{0}^{\tau_{1}} e^{-\lambda s} n\left(x_{s}, A\right) d \sigma_{s}\right) \\
& =E_{x}\left(\int_{0}^{\tau_{1}} e^{-\lambda s} \frac{n\left(x_{s}, A\right)}{n\left(x_{s}, \Gamma_{m}\right)} n\left(x_{s}, \Gamma_{m}\right) d \sigma_{s}\right) \\
& =E_{x}\left(\exp \left(-\lambda \tau_{1}\right) \frac{n\left(x_{\tau_{1}-}, A\right)}{n\left(x_{\tau_{1}-}, \Gamma_{m}\right)} \chi_{\Gamma_{m}}\left(x_{\tau_{1}}\right)\right)
\end{aligned}
$$


by (9.4). Since $\chi_{\Gamma_{m}} \rightarrow 1$ and $n(x, A) \leqq n\left(x, \Gamma_{m}\right)$, we can take the limit and conclude

$$
E_{x}\left(\exp \left(-\lambda \tau_{1}\right) \chi_{A}\left(x_{\tau_{1}}\right)\right)=E_{x}\left(\exp \left(-\lambda \tau_{1}\right) K\left(x_{\tau_{1}-}, A\right)\right)
$$

where $K(x, A)=\lim n(x, A) / n\left(x, \Gamma_{m}\right) \leqq 1$.

Now, by the strong Markov property at $\tau$ (see Theorem 5.4)

$$
T_{t}^{\prime \prime} f(x) \equiv T_{t}^{\circ} f(x)+E_{x}^{\prime \prime}\left[\chi_{[\tau \leqq t]} T_{t-\tau}^{\prime \prime} f\left(y_{\tau}\right)\right]
$$

Taking Laplace transforms both here and in (9.2)

$$
\begin{aligned}
& R_{\lambda}^{\prime \prime} f(x)=R_{\lambda}^{\circ} f(x)+E_{x}^{\prime \prime}\left[e^{-\lambda \tau} R_{\lambda}^{\prime \prime} f\left(y_{\tau}\right)\right], \\
& R_{\lambda}^{\prime \prime} f(x)=R_{\lambda}^{\circ} f(x)+E_{x}\left[\exp \left(-\lambda \tau_{1}\right) R_{\lambda}^{\prime \prime} f\left(x_{\tau_{1}-}\right)\right] .
\end{aligned}
$$

Hence by the pointwise density of the range of $R_{\lambda}^{\prime \prime}=\mathscr{D}\left(A^{\prime \prime}\right)$ in $B_{0}(X)=B_{0}^{\prime \prime}(X)$,

$$
\begin{aligned}
& P_{x}\left(\tau_{1} \leqq t, x_{\tau_{1}-} \in A\right)=P_{x}^{\prime \prime}\left(\tau \leqq t, y_{\tau} \in A\right), \\
& E_{x}\left[\chi_{\left[\tau_{1} \leqq t\right]} K\left(x_{\tau_{1}-}, A\right)\right]=E_{x}^{\prime \prime}\left[\chi_{[\tau \leqq t]} K\left(y_{\tau}, A\right)\right] .
\end{aligned}
$$

Combining (9.5), (9.6) and the relation

yields (9.1).

$$
T_{t} f(x)=T_{t}^{\circ} f(x)+E_{x}\left[x_{\left[\tau_{1} \leqq t\right]} T_{t-\tau_{1}} f\left(x_{\tau_{1}}\right)\right]
$$

For uniqueness, we note that by $\S 4(4.2)$ and (9.1)

$$
A_{\lambda} \subseteq A_{\lambda}^{\prime \prime}\left(I+S_{\lambda}^{\prime \prime}\right)\left(I-S_{\lambda}^{\circ} K\right)
$$

where $S_{\lambda}^{\prime \prime} f(x)=E_{x}^{\prime \prime}\left[\int_{0}^{\infty} e^{-\lambda s} f\left[y_{s}\right] d \phi_{s}\right]$, with (9.7) being attained iff we have uniqueness in (9.1) or iff there exist no bounded Borel solutions of

$$
f(x)=S_{\lambda}^{\circ} K f(x)=E_{x}^{\prime \prime}\left[e^{-\lambda \tau} K f\left(y_{\tau}\right)\right]=E_{x}\left(\exp \left(-\lambda \tau_{1}\right) f\left(x_{\tau_{1}}\right)\right)
$$

again by (9.5), (9.6). However by (4.8)

$$
f(x)=\left(S_{\lambda}^{\circ} K\right)^{n} f(x)=E_{x}\left(\exp \left(-\lambda \tau_{n}\right) f\left(x_{\tau_{n}}\right)\right) \rightarrow 0
$$

since $\tau_{n} \rightarrow \infty$ a.s. Q.E.D. And we have equality in (9.7).

\section{REFERENCES}

1. K. L. Chung, Markov chains with stationary transition probabilities, Die Grundlehren der math. Wissenschaften, Band 104, Springer-Verlag, Berlin and New York, 1960. MR 22 \#7176.

2. E. B. Dynkin, Markov processes, Fizmatgiz, Moscow, 1963; English transl., Vols. I, II, Academic Press, New York and Springer-Verlag, Berlin and New York, 1965. MR 33 \#1886; MR 33 \#1887.

3. W. Feller, On boundaries and lateral conditions for the Kolmogorov differential equations, Ann. of Math. (2) 65 (1957), 527-570. MR 19, 892.

4. A. Friedman, Partial differential equations of parabolic type, Prentice-Hall, Englewood Cliffs, N. J., 1964. MR 31 \#6062.

5. N. Ikeda, M. Nagasawa and S. Watanabe, a) On branching Markov processes, Proc. Japan Acad. 41 (1965), 816-821. MR 34 \#2068.

b) Fundamental equations of branching Markov processes, Proc. Japan Acad. 42 (1966), 252-257. MR 34 \#2069. 
c) A construction of Markov processes by piecing out, Proc. Japan Acad. 42 (1966), 370-375. MR 34 \#2070.

d) A construction of branching Markov processes, Proc. Japan Acad. 42 (1966), 380-384. MR 34 \#2071.

e) Transformation of branching Markov processes, Proc. Japan Acad. 42 (1966), 719-724. MR 35 \#3734.

f) On branching semi-groups. I, II, Proc. Japan Acad. 42 (1966), 1016-1021, 1022-1026. MR 35 \#3735.

6. - Branching Markov processes. I, II, III, J. Math. Kyoto Univ. 8 (1968), 233-278, 365-410; 9 (1969), 95-160. MR. 38 \#764; MR 38 \#6677.

7. K. Itô and H. McKean, Jr., Diffusion processes and their sample paths, Die Grundlehren der math. Wissenschaften, Band 125, Academic Press, New York and Springer-Verlag, Berlin and New York, 1965, Chapter 5. MR 33 \#8031.

8. S. Itô, A boundary value problem of partial differential equations of parabolic type, Duke Math. J. 24 (1957), 299-31L. MR 19, 864.

9. H. McKean, Jr. and H. Tanaka, Additive functionals of the Brownian path, Mem. Coll. Sci. Univ. Kyoto Ser. A Math. 33 (1960/61), 479-506. MR 24 \#A1147.

10. P. Meyer, Fonctionelles multiplicatives et additives de Markov, Ann. Inst. Fourier Grenoble 12 (1962), 123-230.

11. - Processus de Markov, Lecture Notes in Math., no. 26, Springer-Verlag, Berlin and New York, 1967. MR 36 \#2219.

12. M. Motoo, Representation of a certain class of excessive functions and a generator of Markov process, Sci. Papers College Gen. Ed. Univ. Tokyo 12 (1962), 143-159. MR 26 \#7043.

13. J. E. Moyal, Discontinuous Markoff processes, Acta Math. 98 (1957), 221-264. MR $20 \# 344$.

14. M. Nagasawa, Construction of branching Markov processes with age and sign, Kōdai Math. Sem. Rep. 20 (1968), 469-508.

15. M. Nagasawa and T. Sirao, Probabilistic treatment of the blowing up of solutions for a nonlinear integral equation, Trans. Amer. Math. Soc. 139 (1969), 301-310. MR 39 \#736.

16. S. Sawyer, A remark on the S-equation for branching processes, Proc. Japan Acad. (submitted).

17. K. Sato and T. Ueno, Multi-dimensional diffusion and the Markov process on the boundary, J. Math. Kyoto Univ. 4 (1964/65), 529-605. MR 33 \#6702.

18. M. Silverstein, Markov processes with creation of particles, Z. Wahrscheinlichkeitstheorie und Verw. Gebiete 9 (1968), 235-257. MR 37 \#3660.

19. A. V. Skorohod, Branching diffusion processes, Teor. Verojatnost. i Primenen. 9 (1964), 492-497= Theor. Probability Appl. 9 (1964), 492-497. MR 29 \#5295.

20. S. Sobolev, Applications of functional analysis in mathematical physics, Izdat. Leningrad. Gos. Univ., Leningrad, 1950; English transl., Transl. Math. Monographs, vol. 7, Amer. Math. Soc., Providence, R. I., 1963. MR 14, 565; MR 29 \#2624.

21. V. Volkonskiĭ, Additive functionals of Markov processes, Trudy Moskov. Mat. Obšč. 9 (1960), 143-189; English transl., Selected Transl. Math. Stat. and Prob., vol. 5, Amer. Math. Soc., Providence, R. I., 1965, pp. 127-178. MR 25 \#610.

22. S. Watanabe, On discontinuous additive functionals and Lévy measures of a Markov process, Japan. J. Math. 34 (1964), 53-70. MR 32 \#3137.

23. M. Freĭdlin, Diffusion processes with reflection and problems with a directional derivative on a manifold with a boundary, Teor. Verojatnost. i Primenen. 8 (1963), 80-88=Theor. Probability Appl. 8 (1963), 75-83. MR 27 \#810.

24. I. I. Gihman and A. V. Skorohod, Introduction to the theory of random processes, "Nauka", Moscow, 1965; English transl., Saunders, Philadelphia, Pa., 1969. MR 33 \#6689. 
25. V. P. Harlamov, On properties of branching processes with an arbitrary set of particle types, Teor. Verojatnost. i Primenen. 13 (1968), 82-95= Theor. Probability Appl. 13 (1969), 84-98. MR 37 \#3656.

26. T. Sirao, a) A probabilistic treatment of semi-linear parabolic equations, Proc. Japan Acad. 42 (1966), 885-890. MR 36 \#983.

b) On signed branching Markov processes with age, Nagoya Math. J. 32 (1968), 155-225. MR 38 \#768.

\section{BROWN UNIVERSITY,}

Providence, RHOde IsLAND 02912 